\title{
MONODISPERSED POLYGONAL WATER DROPLETS IN MICROCHANNEL
}

\author{
A Thesis \\ by
}

\section{RAJAT MEHROTRA}

\author{
Submitted to the Office of Graduate Studies of \\ Texas A\&M University \\ in partial fulfillment of the requirements for the degree of \\ MASTER OF SCIENCE
}

May 2008

Major Subject: Electrical Engineering 


\title{
MONODISPERSED POLYGONAL WATER DROPLETS IN MICROCHANNEL
}

\author{
A Thesis
}

by

\section{RAJAT MEHROTRA}

\author{
Submitted to the Office of Graduate Studies of \\ Texas A\&M University \\ in partial fulfillment of the requirements for the degree of \\ MASTER OF SCIENCE
}

Approved by:

$\begin{array}{ll}\text { Chair of Committee, } & \text { Jun Kameoka } \\ \text { Committee Members, } & \begin{array}{l}\text { Kuang-An Chang } \\ \text { Deepa Kundur }\end{array} \\ & \begin{array}{l}\text { Chin Bing Su } \\ \text { Head of Department, }\end{array}\end{array}$

May 2008

Major Subject: Electrical Engineering 


\begin{abstract}
Monodispersed Polygonal Water Droplets in Microchannel. (May 2008)

Rajat Mehrotra, B.S., Texas A\&M University

Chair of Advisory Committee: Dr. Jun Kameoka
\end{abstract}

The fabrication, motion and behavior of small droplets are subjects under considerable current study. The possible applications include using droplets as actuators to enhance mixing, as chemical reactors and the formation of emulsions. Microfluidics provides a convenient means of producing droplets at the micro scale. The study is currently dominated by spherical systems where droplets are consistently spherical in nature. Various methods and geometries have been tested for fabricating these droplets but little research has been conducted towards producing non-circular droplets. While the fabrication of non-spherical droplets has been reported before control over their shape remains difficult to achieve.

In this thesis, we present a method to fabricate droplets using shear focusing in an oil medium alternatively from two channels facing each other. The droplets produced are non-circular in shape, and their shape dynamically alters as they travel in the microfluidic channel. The size of the droplets can be controlled by the ratio of oil and water flow rates.

Microscopic images have been presented that show the non-spherical shape of the droplets at the point of fabrication. Images taken at two points further along the 
microfluidic channel show how the shapes of these droplets change as they travel in the channel. There were three regimes of droplet shapes, circular, triangular and rectangular shapes that were determined by the packing ratio of water droplet in oil phase in microfluidic channels. All droplets formed in this experiment were monodispersed. 
To my parents and my sister. 


\section{ACKNOWLEDGEMENTS}

I would like to thank my committee chair, Dr. Kameoka, and my committee members, Dr. Su, Dr. Kundur and Dr. Chang, for their guidance and support throughout the course of this research.

Thanks also go to my friends and colleagues and the department faculty and staff for making my time at Texas A\&M University a great experience.

Finally, thanks to my mother, father and sister for their encouragement. 


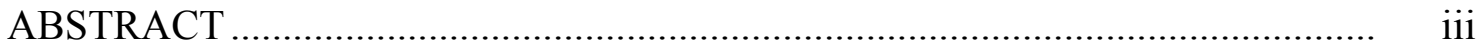

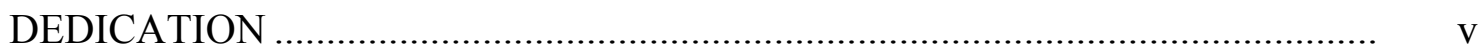

ACKNOWLEDGEMENTS ………………………….................................. vi

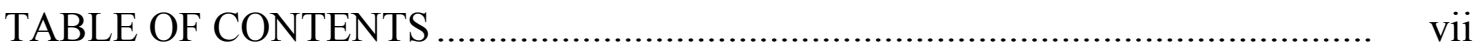

LIST OF FIGURES .................................................................................. ix CHAPTER

I INTRODUCTION: MICROFLUIDICS.......................... 1

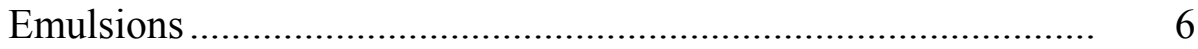

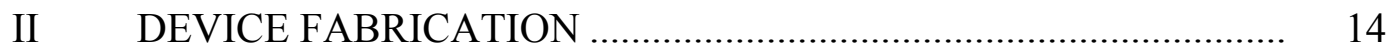

Bottom-Up Approach..................................... 14

Top-Down Approach......................................... 16

III DEVICE DESIGN............................................ 22

T Channel................................................. 22

Cross Channel..................................................... 24

Cross Channel with Shear Force............................... $\quad 25$

IV FORMATION OF NON-SPHERICAL DROPLETS AT CROSS

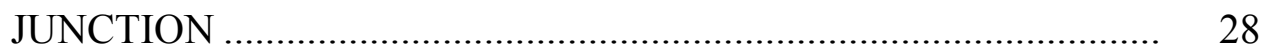

$\mathrm{V} \quad$ DYNAMIC SHAPE MODIFICATION OF DROPLETS .................... 33 
CHAPTER Page

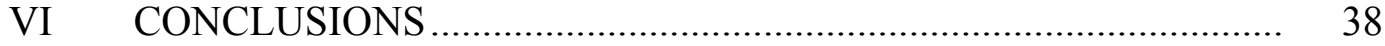

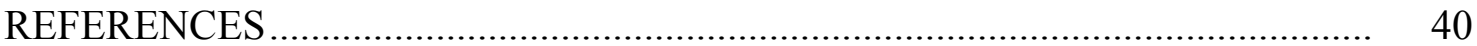

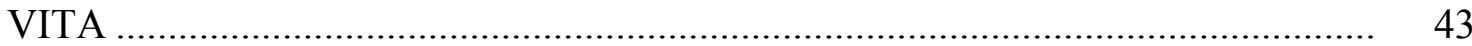




\section{LIST OF FIGURES}

FIGURE Page

1.1 Flow patterns of laminar flow and turbulent flow.................................. 2

1.2 Photographic images of microfluidic devices .................................... 4

$1.3 \quad$ Lab-on-a-chip example ................................................................. 5

1.4 Droplets being produced in a microfluidic channel ................................ 6

1.5 Applications of emulsions: Emulsions being used to enhance mixing in

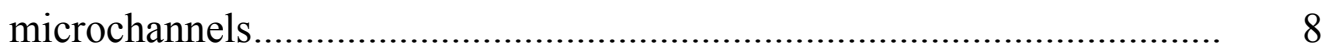

1.6 Applications of emulsions: Emulsions being used to create a Ring Oscillator with 3 AND gates ......................................................... 9

1.7 Shape controlled microparticles produced by using continuous flow lithography................................................. 11

1.8 Non-spherical colloids........................................ 12

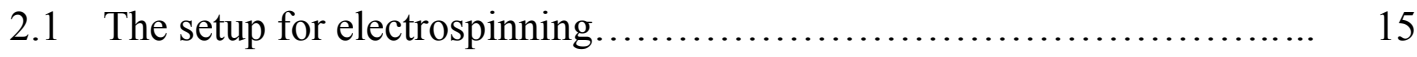

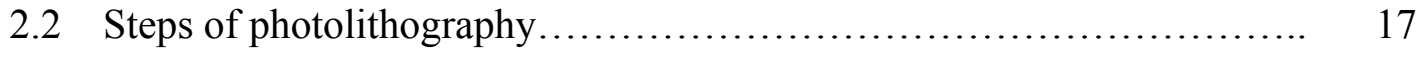

2.3 Fabrication of microfluidic devices using polydimethylsiloxane

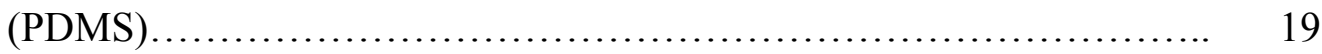

2.4 Experimental setup for microfluidic experiment.................... 20

$3.1 \quad \mathrm{~T}$ channel.................................................. 23

3.2 Cross channel.................................................... 24 
FIGURE Page

3.3 a) Schematic diagram of the microchannel device. Water flows in from inlet 1 and oil from inlet 2. b) Microscopic image of area enclosed within dashed rectangle from figure 3.3.a. $\mathrm{W}=50 \mu \mathrm{m}, \mathrm{D} 1=15 \mu \mathrm{m}, \mathrm{D} 2=30$ $\mu \mathrm{m}, \mathrm{D} 3=30 \mu \mathrm{m} \mathrm{H}=100 \mu \mathrm{m}$. The depth of the channels is $50 \mu \mathrm{m} \ldots \ldots \ldots . .26$

4.1 Formation of droplets at the cross junction (detection point A)......... 28

4.2 Path of droplets in microchannel............................................. 29

4.3 Parabolic flow profile of microchannel........................... 30

4.4 Microscopic images of the cross junction (point A).................. 31

5.1 Microscopic images of microchannel at detection points B and C at various flow rates.............................................

5.2 Water droplet packing ratio in oil media and displacement between droplets as the function of water/oil flow rate ratio.

5.3 Microscopic images of microchannel at detection point $\mathrm{C}$ at various flow rates 


\section{CHAPTER I}

\section{INTRODUCTION: MICROFLUIDICS}

Microfluidics is a study that deals with the control and manipulation of fluids in devices where significant lengths are less than a millimeter in size. Interest in microfluidics has been steadily increasing with the availability of many new fabrication techniques now available that make it possible to build such small devices with great precision and the advancements made in biotechnology that make it possible to detect and manipulate at the micron scale. Many disciplines, such as physics, chemistry, and multiple engineering fields have recognized the potential of microfluidics and are applying it in their research studies.

The development of microfluidics can be traced back to the emergence of sophisticated fabrication techniques in the 1950s. These techniques gave birth to the field of microtechnology which has since then grown at an astonishing rate. Another important field that developed with the help of these techniques was microelectromechnical systems (MEMS). This involved building mechanical structures at the micron scale and was directly responsible for emergence of microfluidics. Microfluidics combined the properties of MEMS with fluidics. The fabrication techniques used to develop these small devices continues to improve to this day and have now given us the ability to make channels at the nanometer scale giving birth to a new field of research know as nanofluidics.

This thesis follows the style of Lab on a Chip. 
Fluids behave differently at the micro scale as compared to the macro scale. Factors such as viscosity and surface tension start dominating the system. A key difference is that the Reynolds number $(\mathrm{Re})$ at the micro scale is much smaller. In fluid dynamics the Reynolds number is defined as the ratio of inertial forces to viscous forces and is used to predict whether the flow is turbulent or laminar. In the case of microfluidic channels the flow tends to be laminar $(\operatorname{Re}<=1)$. Thus, two fluids flowing next to each other do not readily mix besides diffusion at the interface. Figure 1.1 gives a graphic description of the difference between the two flows. Other properties such as surface tension and fluidic resistance also differ at this scale. Microfluidics seeks to take advantage of these changes in properties or work around them to accomplish a task.

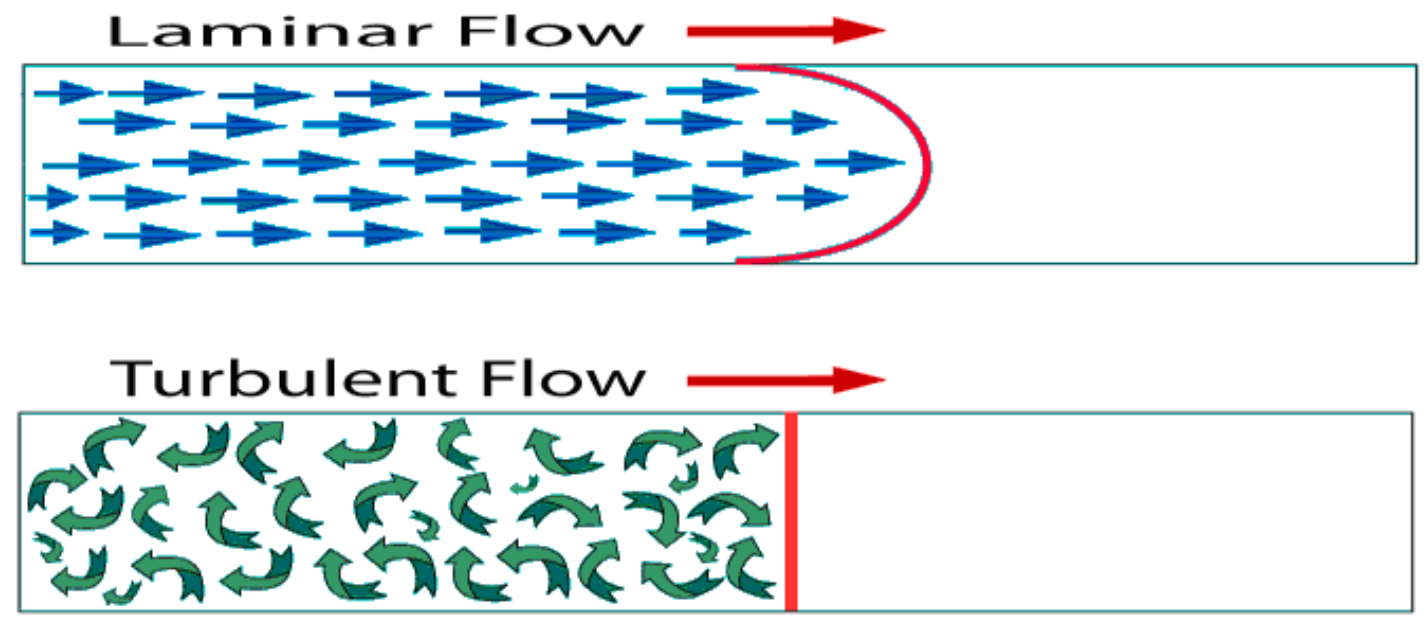

Fig. 1.1 Flow patterns of laminar flow and turbulent flow. Laminar flow is seen in microchannels and turbulent flow in marcochannels. 
Microfluidics' greatest attraction has been recognized as its ability to work with extremely small structures. This makes it very suitable for medical studies and for dealing with cellular matter. Microfluidics is currently being used to study single cell ${ }^{1}$ and single molecule biophysics ${ }^{2}$. A great amount of research work is currently being done towards producing cheap and accurate lab-on-a-chip devices that may be used as diagnostic tools or for other analytical tasks ${ }^{3}$. Some of the other advantages that microfluidics offers include portability due to the small size of the devices and cost savings by using small volumes of reagents (typically only milliliters of volumes are required to conduct experiments at the micro scale). It also offers quick results and potential for highly parallel analysis.

Microfluidics has already been applied to produce results in various fields. These include DNA and protein analysis ${ }^{4-5}$, biochemical toxins detection ${ }^{6}$ and drug delivery ${ }^{7}$ and discovery ${ }^{8}$.

Photographic images of various microfluidic devices are shown in figure 1.2. Figure 1.3 gives a detailed schematic view of a sample lab-on-a-chip device. 

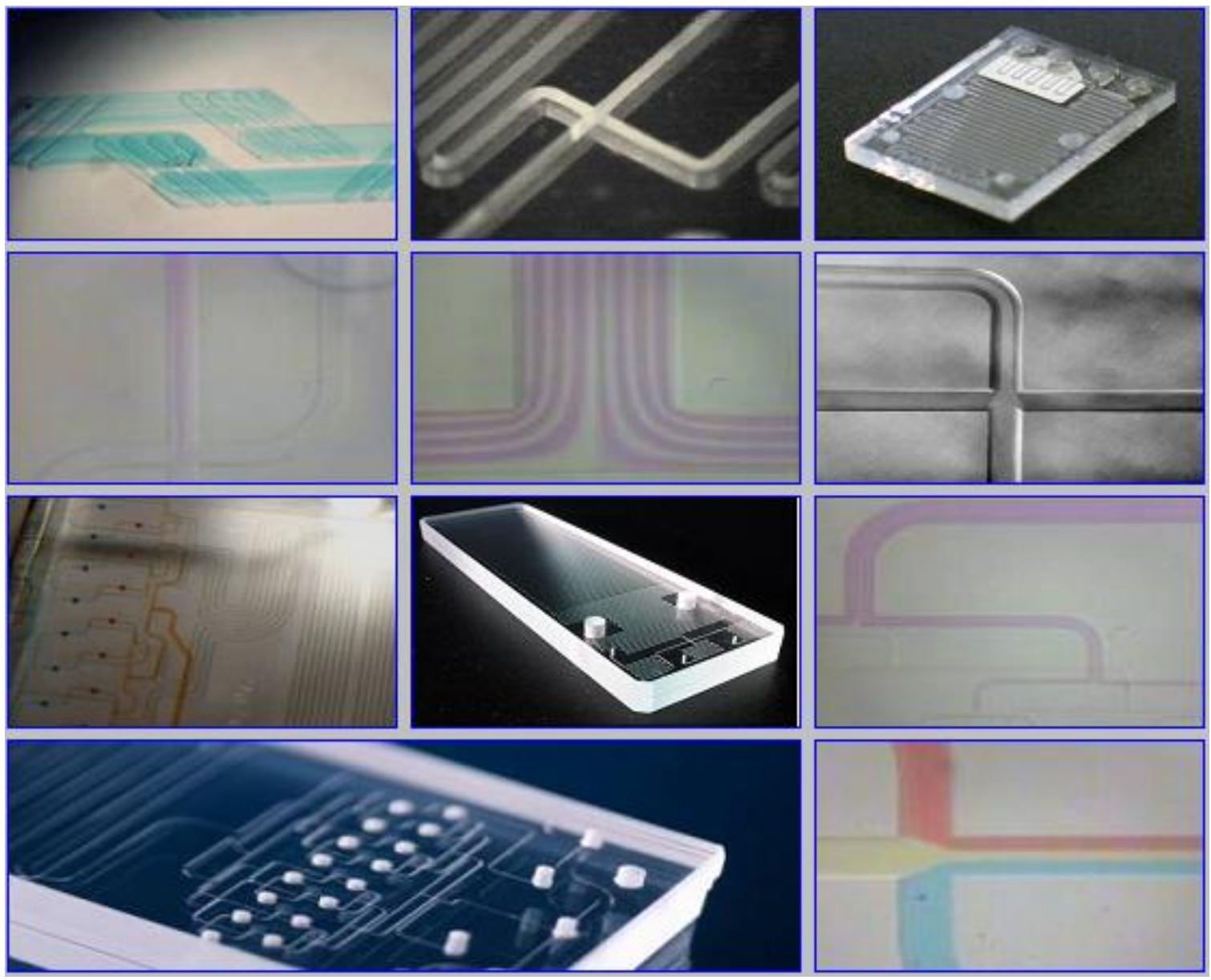

Fig. 1.2 Photographic images of microfluidic devices. 

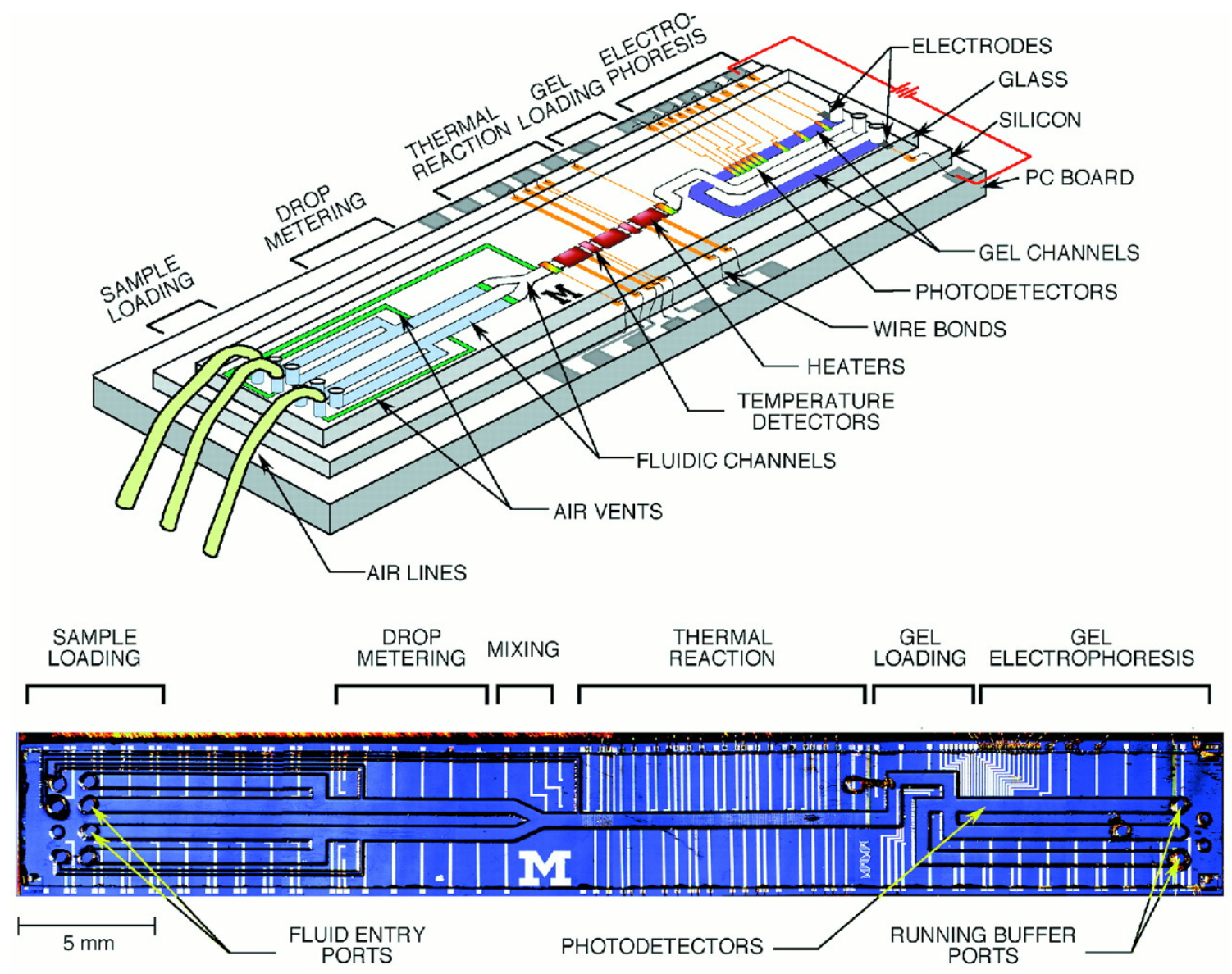

Fig. 1.3 Lab-on-a-chip example 9 


\section{Emulsions}

Emulsions are systems of two immiscible liquids. In these systems droplets of liquids are dispersed in to another liquid. The first liquid is called the dispersed phase and the second liquid, in which the droplets are formed, is called the continuous phase. Emulsions are commonly seen in daily life and milk and butter are good examples of that. Butter has a lipid phase in which water droplets are dispersed while milk is an emulsion of fat droplets in a mixture of water, sugar and proteins.

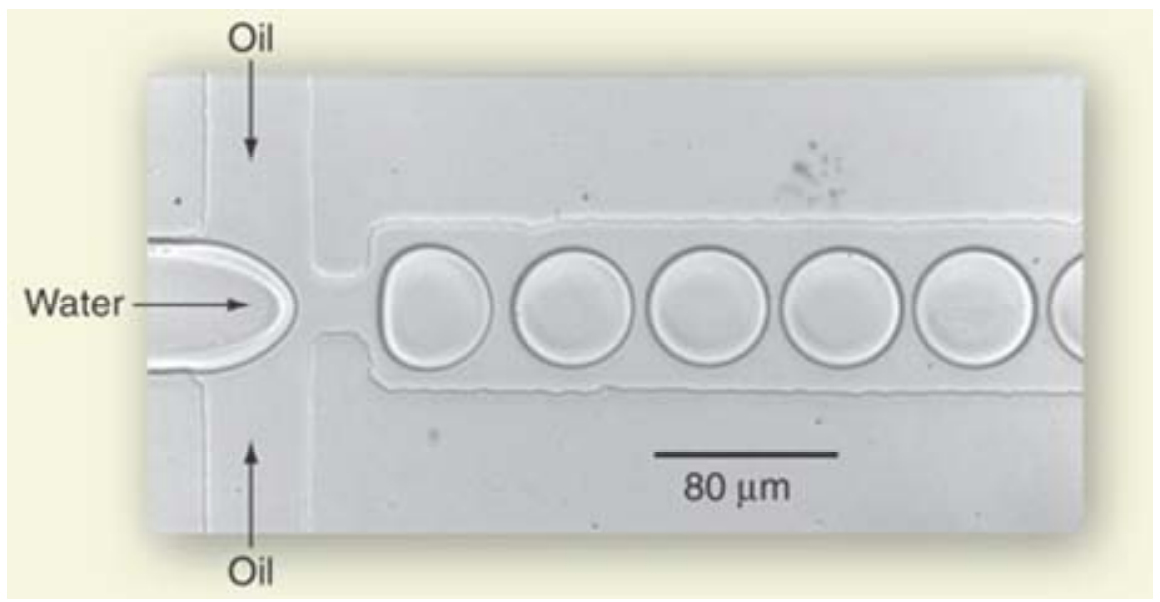

Fig. 1.4 Droplets being produced in a microfluidic channel ${ }^{10}$.

Figure 1.4 is an example of how droplets are produced in microfluidic channels. In the channel water droplets are bring produced in an oil medium, hence water is the dispersed phase and oil is the continuous phase. The droplets are spherical in shape. The size of these droplets can be controlled by controlling the flow rates of the two media. 
Water and oil and the two most commonly used media to produce droplets due to the neutral nature of water.

Droplet based microfluidics is based on working with these discrete droplets in microfluidic devices. This method of generating uniform and steady emulsions has many potential applications in modern medicine and science ${ }^{11}$. Droplets can be produced by various methods using a number of geometries and fluids. The droplets create controlled conditions and are ideal for use as chemical reactors. Water droplets have been used as chemical reactors as they provide an isolated environment to conduct chemical reactions $i^{12}$. A second application includes producing microparticles by using a UV-curable polymer to produce the droplet. Once the droplet is formed it is exposed to UV light and it polymerizes to produce a microparticle ${ }^{13}$. By coupling the device with Raman spectroscopy the chemistry of a reaction can be studied at any point in the channel. By changing initial conditions of droplet formation its effect on the reaction can be studied $^{14}$. Due to the low Reynolds numbers of microchannels fluids tend to not mix with each other. Emulsions are one of the many methods employed to enhance mixing in these channels ${ }^{15}$. Figure 1.5 shows one of these methods. Multiple emulsions of waterin-oil-in-water can be formed by encapsulating one droplet into another ${ }^{16}$. To do so, the surface properties of the channels have to be altered; water droplets are formed in a hydrophobic channel in a stream of hydrophobic fluid. Several other useful tools based on droplets have already been developed for tasks such as, fluidic transport ${ }^{17}$ and bioassays ${ }^{18}$, protein crystallization ${ }^{19}$ and drug delivery ${ }^{7}$. Figure 1.6 shows another unique application of microfluidics where emulsions are being used to carry information. 


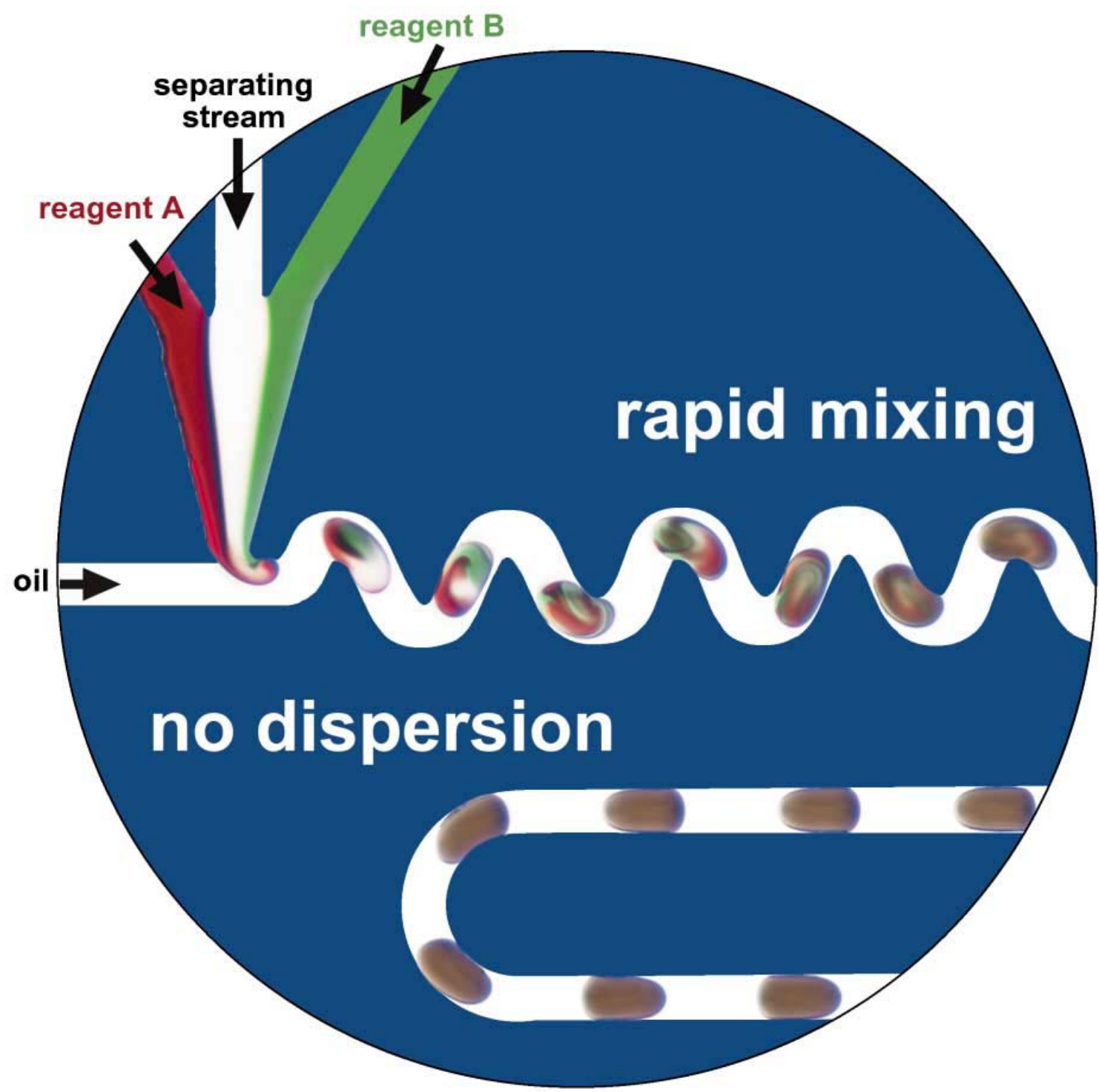

Fig. 1.5 Applications of emulsions: Emulsions being used to enhance mixing in microchannels. The fluids to be mixed are introduced in to a single droplet and rapid mixing takes place as that droplet travels within a channel ${ }^{12}$. 


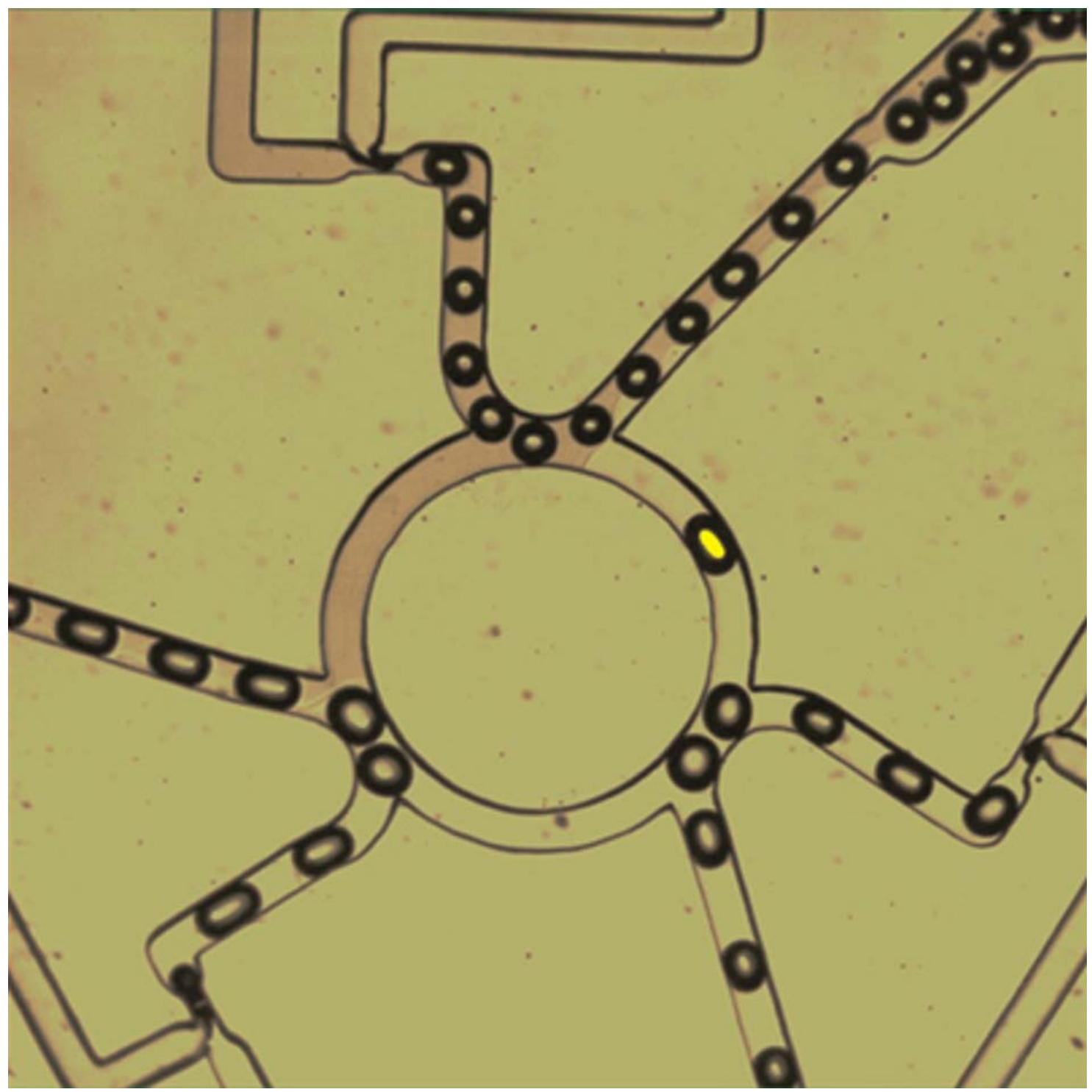

Fig. 1.6 Applications of emulsions: Emulsions being used to create a Ring Oscillator with 3 AND gates. The presence or absence of a droplet in a channel is seen as a piece of information $(0 \text { or } 1)^{20}$. 
The formation of nanoliter and microliter droplets of uniform size in microfluidic channels has been reported before and has biological and chemical applications such as the ones listed above ${ }^{21}$. While the size of these droplets is easy to control by controlling the flow rates of the two media, the control of droplets' shape has proven to be a hurdle. The study of these droplets has so far been dominated by spherical systems. This is because droplets tend to adapt to a spherical shape by nature. This phenomenon can be seen in everyday life, for example water droplets falling from a tap would tend to be roughly spherical. The spherical shape has a unique property; the surface to volume ratio is smallest for a given volume if the volume is spherical in shape. Thus the interfacial surface energy is least for a sphere and hence all droplets tend to adapt to this shape. Control over the shapes of droplets in microfluidic channels has proven to be a difficult task.

However, non-spherical microparticles have also been produced before. Dendukuri et al produced non-spherical microparticles (see figure 1.8) using a T-channel and confining them using appropriate microchannel geometries ${ }^{13}$. Nisisako and Torii were able to synthesize shape-controlled microparticles using flow focusing ${ }^{22}$. Researches have been able to combine the properties of microfluidics and photolithography to develop a technique called continuous flow lithography. Doyle and colleagues at the Massachusetts Institute of Technology have devised a new technique called continuous-flow lithography (see figure 1.7), that combines photolithography and microfluidics to synthesize microparticles of various shapes ${ }^{23}$. 

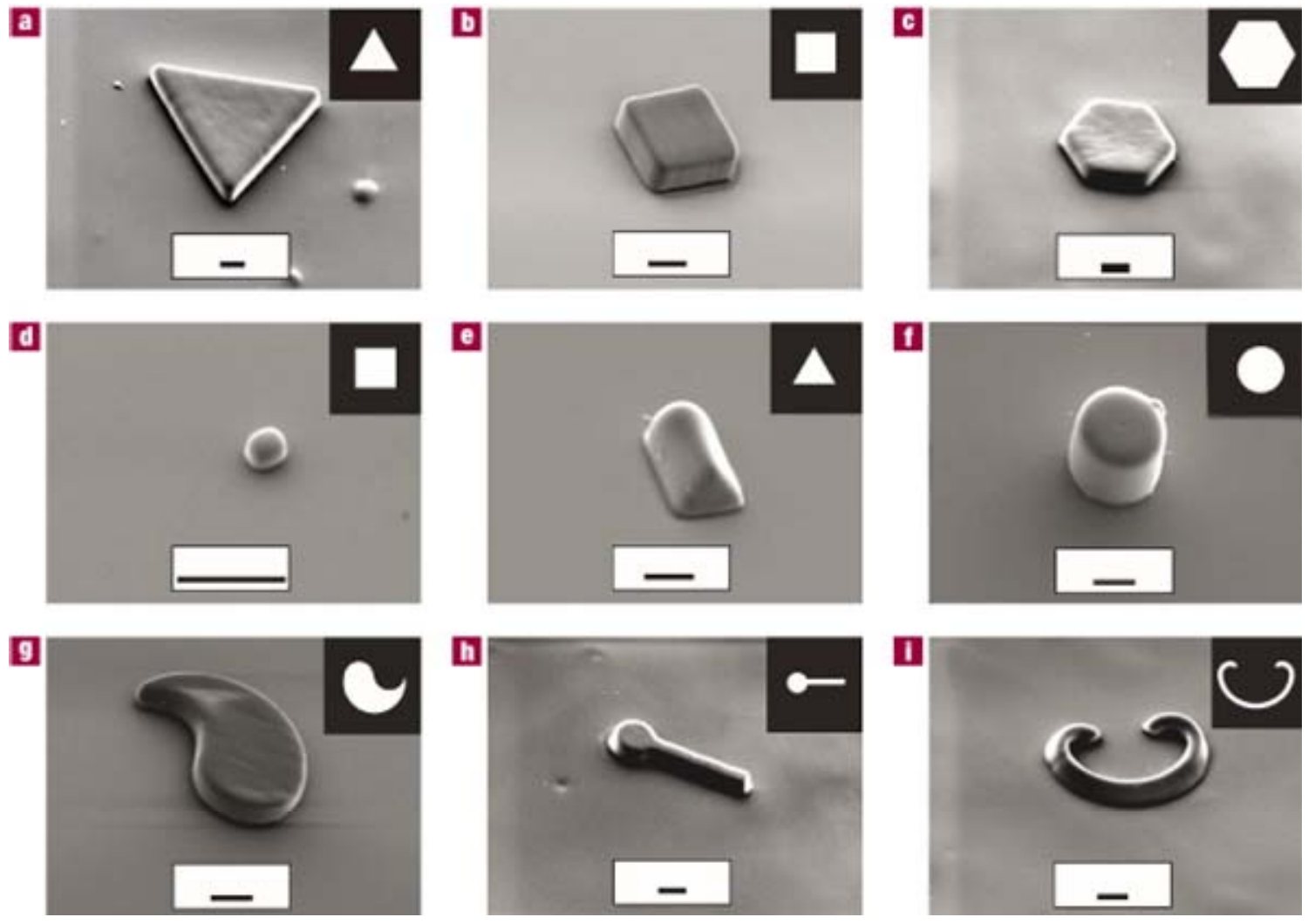

Fig. 1.7 Shape controlled microparticles produced by using continuous flow lithography ${ }^{23}$. 

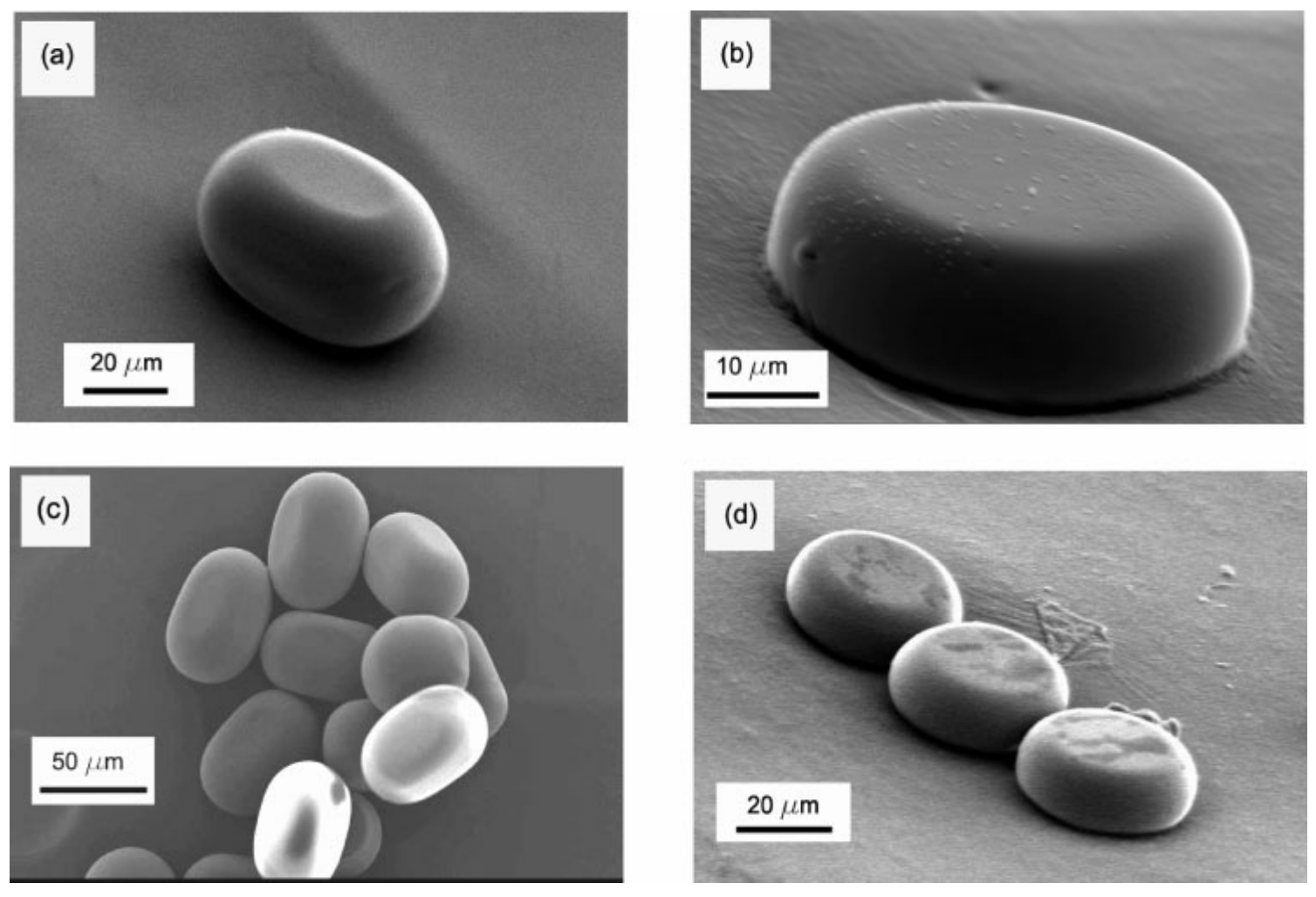

Fig. 1.8 Non-spherical colloids ${ }^{13}$

Despite the above listed research experiments carried out for the formation of non-spherical droplets and particles the study of micro and nanoliter droplets has so far been dominated by spherical systems. The properties of non-spherical droplets could be used for a number of applications. They can be used to form non-spherical colloids such as disks and rods or other unique structures or to test response to external fields. Nonspherical colloids can be used as building blocks to build other structures. Yet, control over the shape of microdroplets remains difficult to achieve and the dynamic modification of droplet shape has not been reported before. 
In this thesis we describe a novel application of microfluidics to create nonspherical droplets. We have used a cross channel design that alternatively produces droplets from two channels that face each other. . This way, the packing ratio of water droplet in oil phase is significantly increased by comparing to the droplet formation based on single $\mathrm{T}$ junction. The shearing flow technique has been used to create droplets. Size of the droplets is controlled by changing the flow parameters. The droplets are observed to dynamically change their shape as they travel in the microfluidic channel. These changes are observed at various flow rates. The change in shape is not significant for small droplets but the as the size increases the change become more prominent. Droplets have been observed at three different points within the microfluidic channel to show the dynamic nature of the change in shape. Optical microscope images of droplets have been used in this paper to show these dynamic changes. The dynamic shape modification of droplets has not been reported previously. Depending upon channel lengths, widths and geometries it is possible to generate droplets of varying shapes and sizes. 


\section{CHAPTER II}

\section{DEVICE FABRICATION}

There are two approaches to building microfluidic devices. These can broadly be classified as the Bottom-Up Approach and the Top-Down Approach.

\section{Bottom-Up Approach}

The bottom-up approach relies on the self assembly of smaller parts. This method is used to build structures such as wires and tubes at the nanometer scale. This approach utilizes the self assembly of molecules under certain conditions to build the desired structure. A good example of the bottom-up approach is electrospinning.

Electrospinning uses electrical charge to produce very fine fibers. The setup for electrospinning, as shown in figure 2.1, consists of a metallic needle, a syringe pump, a grounded collector and a power supply. The syringe is filled with a polymer solution and pushed until a drop forms at the needle tip. When voltage is applied to the needle the drop stretches in to what is known as the Taylor Cone. This cone then releases a liquid jet which is deposited on the collector. This thin jet quickly polymerizes to form wires.

The bottom-up approach offers a number of advantages. Structures produced are highly reproducible, thermodynamically stable and economical. 


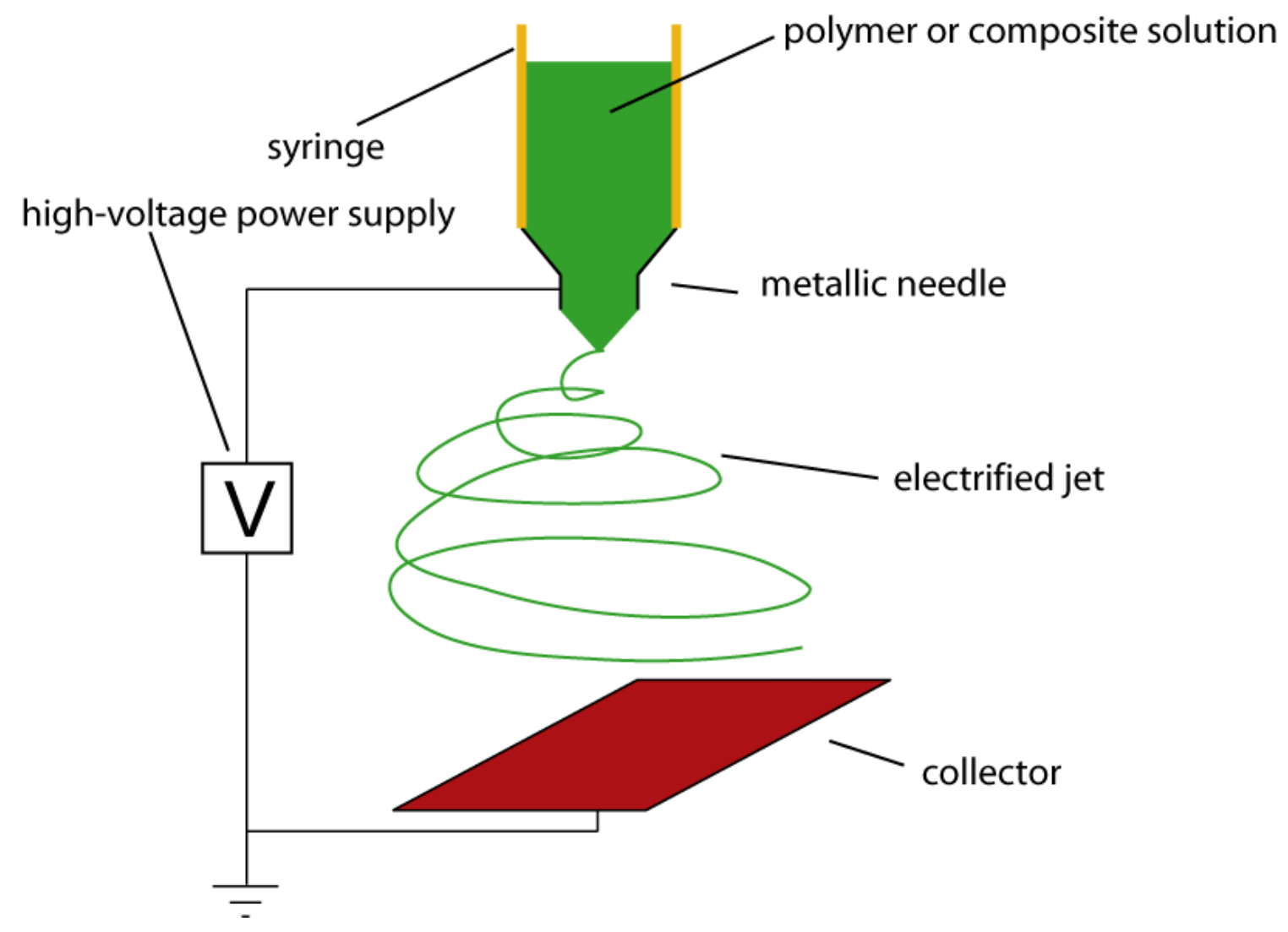

Fig. 2.1 The setup for electrospinning. 


\section{Top-Down Approach}

A more commonly used method is the top-down method. This method relies on selectively removing parts of a thin film to get the desired structure. A number of techniques such as photolithography, E-beam lithography and plasma etching are employed to make devices by this method. Even though this approach is costlier it is more popularly used because it gives you better control.

Photolithography is a commonly used technique used to build microfluidic devices. This is also the method employed in this experiment.

The process of photolithography begins by creating the design of the device. This design is transferred to a mask. Once the mask is ready the design is copied to a silicon wafer. This process involves several steps. To begin with, a silicon wafer is spin coated with a photoresist. The wafer is then soft baked to harden the photoresist. After soft baking, the wafer is placed on a mask aligner under the correct mask and exposed to UV light. For a positive photoresist the exposed parts become less stable while for a negative photoresist the exposed parts become more stable. The wafer is then hard baked and finally developed by immersing it in a developing solution. The less stable parts of the photoresist are washed away and in this manner the design of the mask is transferred to the wafer. This silicon wafer with the device design on it is known as the 'master'. Figure 2.2 gives a step by step illustration of the entire process. 

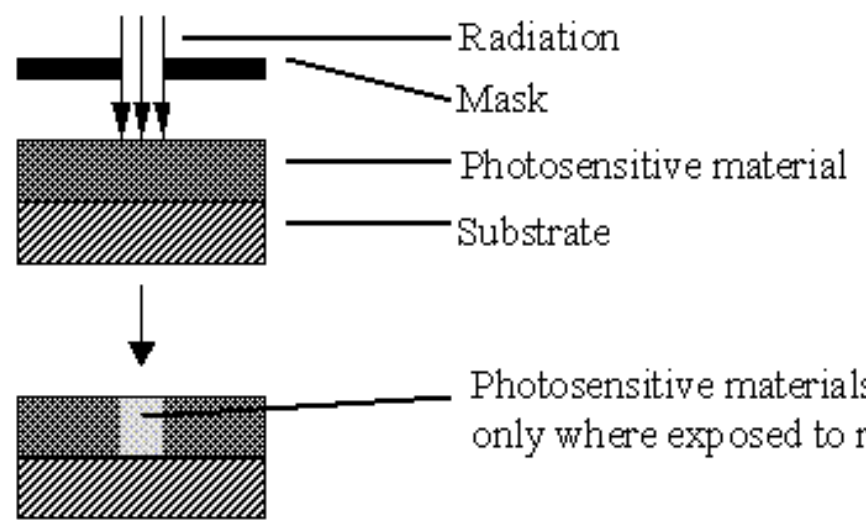

Photosensitive materials properties change only where exposed to radiation

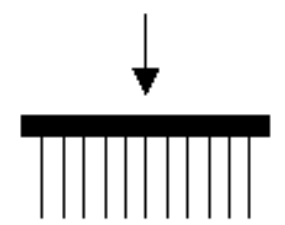

Spray substrate with

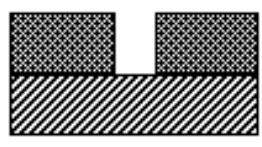

a) Positive resist, developer solution removes exposed material

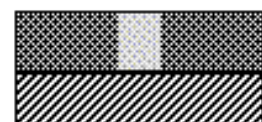

\section{developer solution}

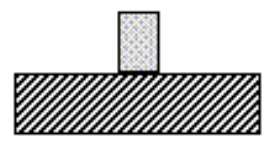

b) Negative resist, developer solution removes unexposed material

Fig. 2.2 Steps of photolithography. 
To make a microfluidic device using a silicon master we have used a silicon polymer known as polydimethylsiloxane. Polydimethylsiloxane (PDMS), a silicon based organic polymer, with the chemical formula $\left(\mathrm{H}_{3} \mathrm{C}\right)_{3}\left[\mathrm{Si}\left(\mathrm{CH}_{3}\right)_{2} \mathrm{O}\right]_{\mathrm{n}} \mathrm{Si}\left(\mathrm{CH}_{3}\right)_{3}$ is the most widely used material used for fabrication of microfluidic devices. Its chemically inert, long durability and optically transparent properties make it highly suitable for this application. PDMS is thoroughly mixed with a silicon elastomer curing agent and poured over the silicon substrate. The ratio used for PDMS and the elastomer is 10:1 by weight. The mixture is allowed to harden by placing it in an oven. This can take up to 2 hours at a temperature of $75^{\circ} \mathrm{C}$. The solid PDMS with the substrate pattern is then peeled off and bonded with a glass substrate using reactive ion etching to form the base. Figure 2.3 gives a step by step illustration of the entire process.

For this experiment the silicon substrate was pre-prepared by photolithography. UV light is used to transfer a pattern from photomask to a silicon wafer coated with a negative photoresist which is then chemically treated.

This fairly simple and inexpensive method was used to create the microfluidic device for this experiment. A second layer of PDMS is poured that holds tubes in place. The tubes are placed at the appropriate positions for inlets and outlets of the device. These tubes are used to transport fluids to the device. 

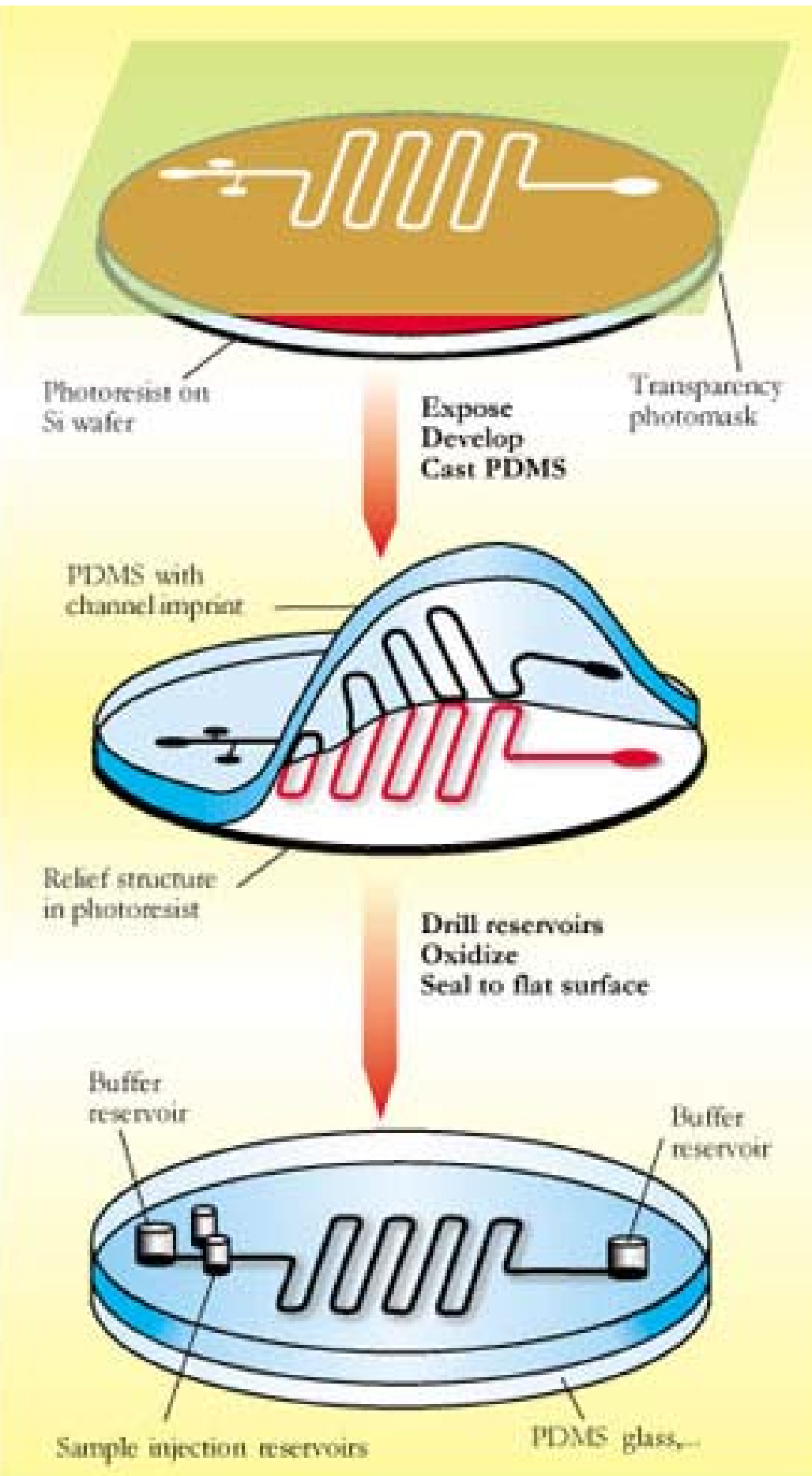

Fig. 2.3 Fabrication of microfluidic devices using polydimethylsiloxane (PDMS) ${ }^{24}$. 
Once the device is ready for the experiment the tubes connected to the inlets and outlets are attached to syringes. These syringes are pre-filled with the fluids that will be used in the experiment. For our experiment we used olive oil with 2\% SPAM 80 surfactant, and water. The surfactant is added to oil to ensure that water droplets that get too close to each other to do not merge. Syringe pumps (Harvard Apparatus) are used to push the liquid in to the device. The flow rate of the fluids can be externally controlled by these pumps.

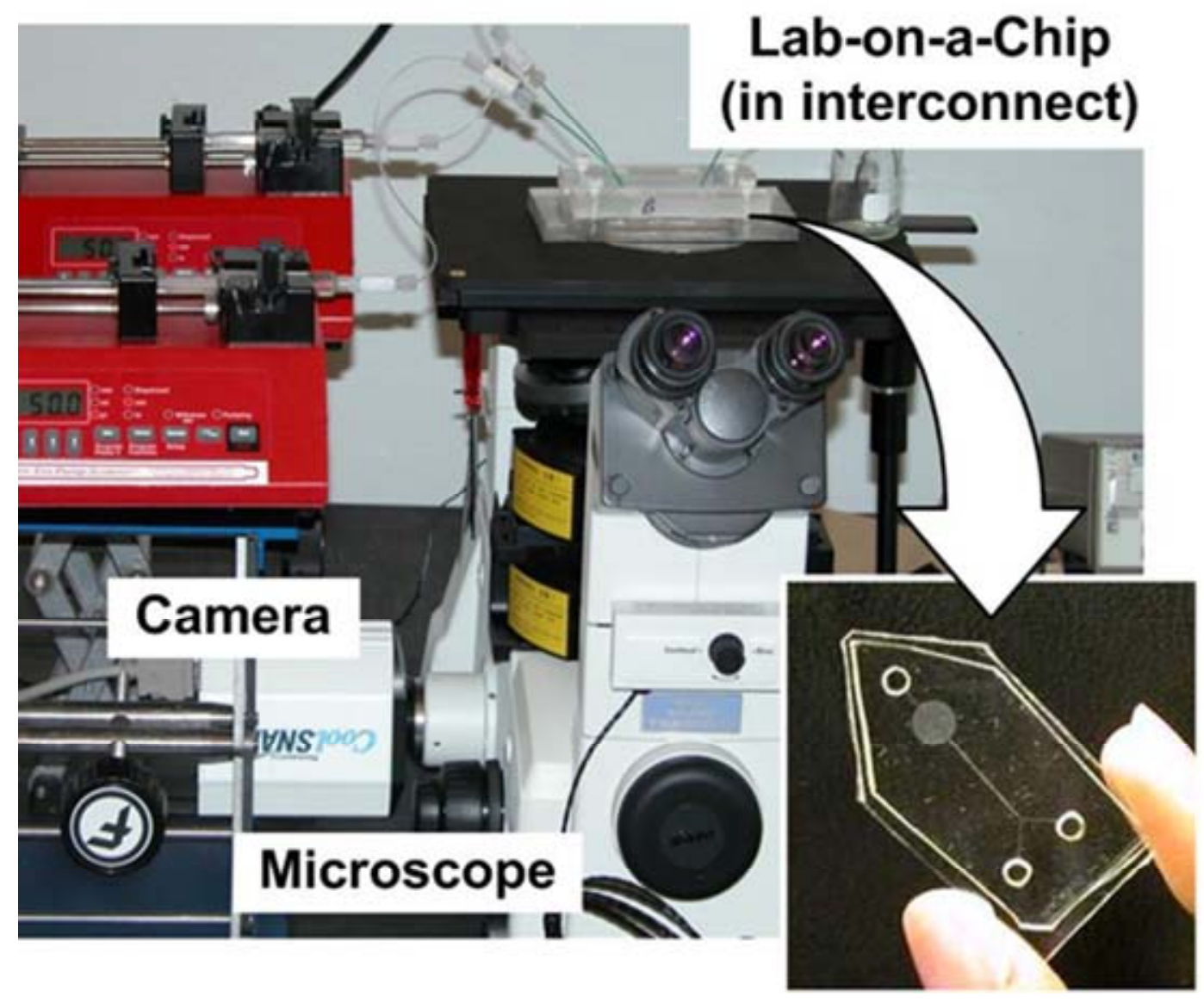

Fig. 2.4 Experimental setup for microfluidic experiment ${ }^{25}$. 
For the setup of the experiment the microfluidic device is placed on a microscope's stand. Since both glass and PDMS are transparent it is easy to view the channels through the microscope. The tubes are connected to the syringe pumps via syringes. Results are recorded using a high speed camera. This set up is shown in the photographic image of figure 2.4. For this experiment we used a Phantom v5.1 camera. The exposure time to take images was set to $1 / 4000 \mathrm{sec}$. 


\title{
CHAPTER III
}

\section{DEVICE DESIGN}

\begin{abstract}
While there are several geometries ${ }^{26}$ that have been used in the past to create droplets in microfluidic experiments, there are two designs that most commonly used, the T channel ${ }^{27-29}$ and the Cross Channel ${ }^{30-32}$.
\end{abstract}

\section{T Channel}

The $\mathrm{T}$ shaped channel is an arrangement of two channels lying perpendicular to each other as shown in figure 3.1.

The two immiscible liquids are pumped in to the two channels. The continuous phase (usually oil) breaks the flow of the dispersed phase (usually water) to create droplets at the junction of the two channels. The force applied by oil at the point of creation is known as shear force ${ }^{33}$. The size of the droplets can be controlled by controlling the flow rates of the two liquids. 


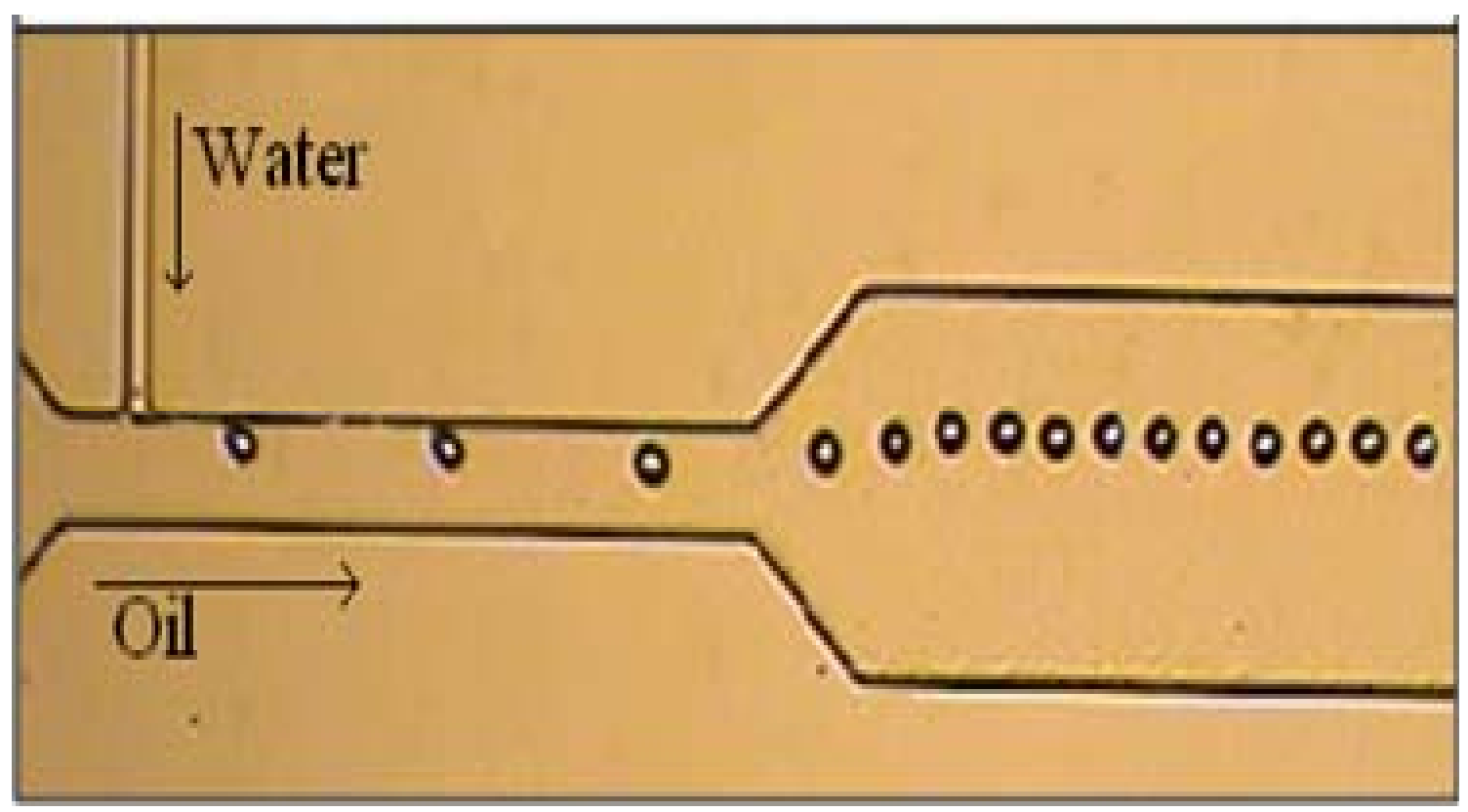

Fig. 3.1 $\mathrm{T}$ channel 


\section{Cross Channel}

The cross channel geometry is an arrangement of microfluidics channels in the shape of a cross as shown in figure 3.2.

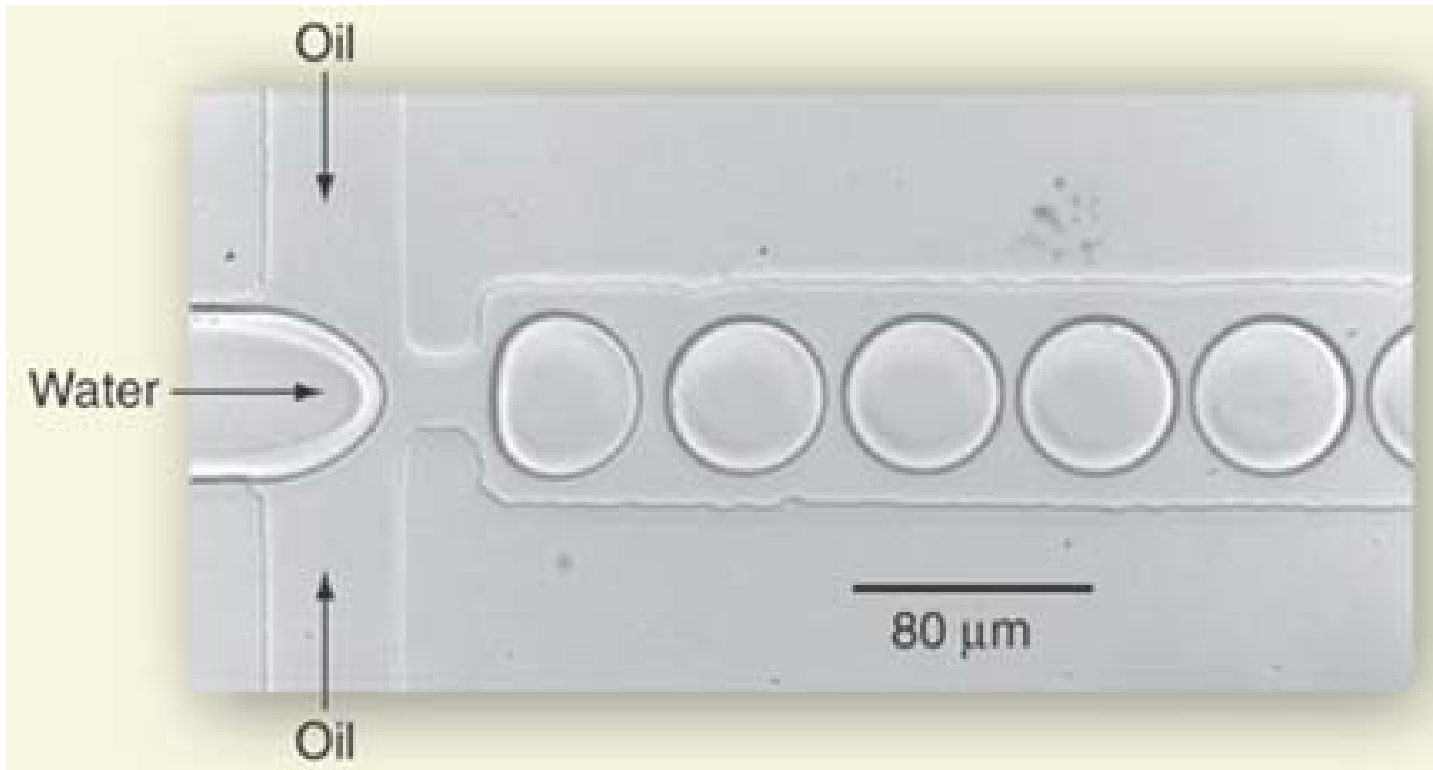

Fig. 3.2 Cross channel

The continuous phase flows in from two channels lying horizontally opposite to each other. The dispersed phase meets the two channels at the junction where applied pressure from the two oil channels focuses the water flow to a cone that releases droplets periodically. This technique is known as flow focusing ${ }^{34}$ and is commonly applied in microfluidic experiments. 


\section{Cross Channel with Shear Force}

For the purpose of this experiment we have combined the properties of the cross channel and the $\mathrm{T}$ channel geometries. The device uses the cross channel geometry but uses shear force to create droplets. We have achieved this by switching the flow of oil and water.

Traditionally this device has been used to generate water droplets in an oil medium using the flow focusing method. In our experiment, we've used this device to generate droplets using shear focusing by switching the water and oil inlets. Water is pumped from one inlet and the channel breaks up into two separate channels which travel around the oil channel to meet at a cross shaped junction. The two water channels lie opposite to each other; the oil is pumped in from the left and the outlet channel is placed at the right end of the cross junction. The water flow is cut off by the oil flow by shear force and droplets are generated at periodic intervals. This design can also be seen as a double ended $\mathrm{T}$ channel.

This double ended $\mathrm{T}$ channel gives us some interesting properties. The applied shear force is twice that of the single $\mathrm{T}$ channel. With droplets being produced from two channels the production rate of this device is almost two times that of the conventional $\mathrm{T}$ channel. Due to this faster production rate distance between consecutive droplets is much smaller compared to a single $\mathrm{T}$ channel. This gives the droplets the chance to influence each others' shapes. This change has been studied in this experiment. 

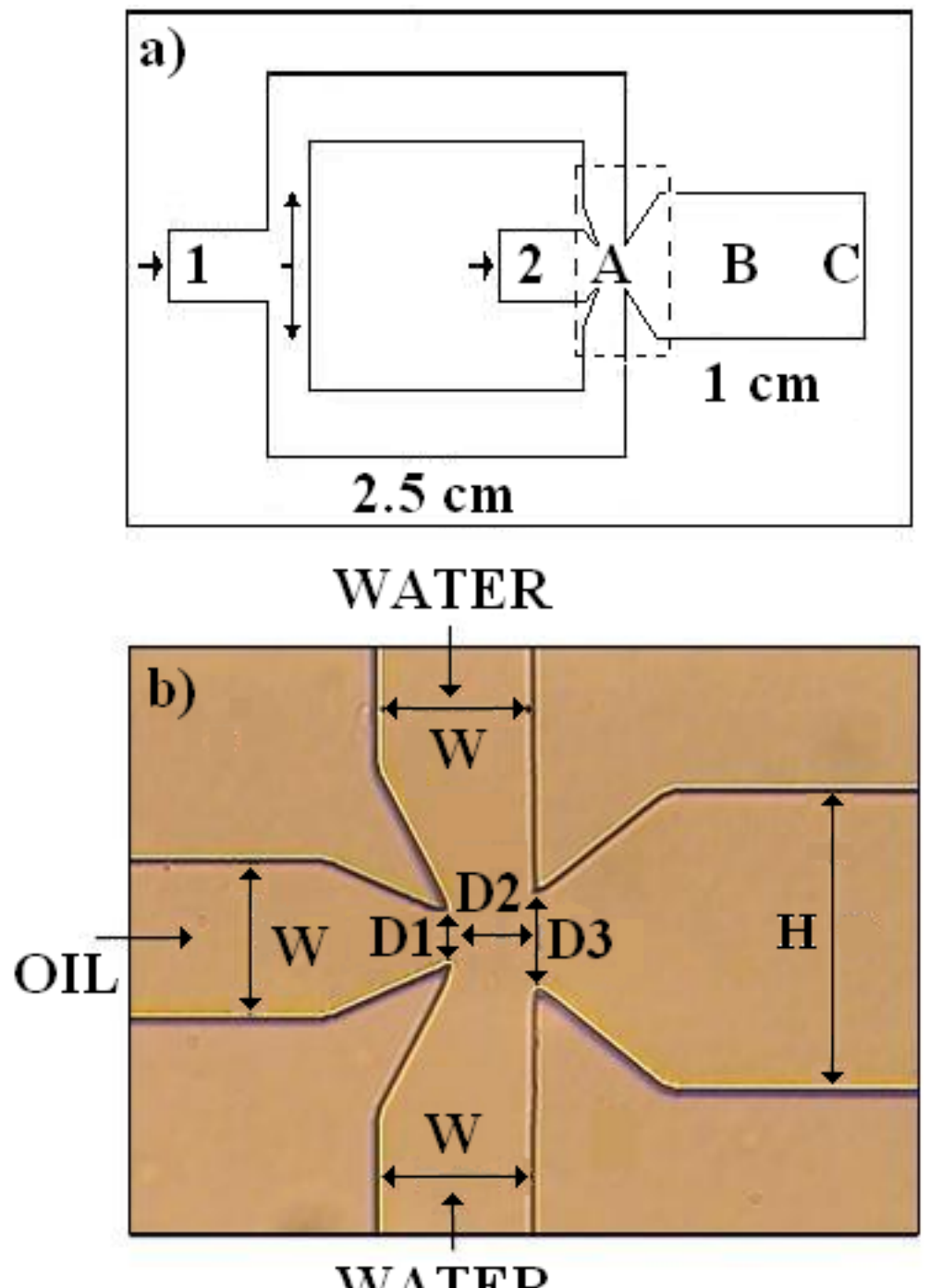

WATER

Fig. 3.3 a) Schematic diagram of the microchannel device. Water flows in from inlet 1 and oil from inlet 2. b) Microscopic image of area enclosed within dashed rectangle from figure 3.3.a. $\mathrm{W}=50 \mu \mathrm{m}, \mathrm{D} 1=15 \mu \mathrm{m}, \mathrm{D} 2=30 \mu \mathrm{m}, \mathrm{D} 3=30 \mu \mathrm{m} \mathrm{H}=100 \mu \mathrm{m}$. The depth of the channels is $50 \mu \mathrm{m}$. 
The dimensions of channels are shown in Fig. 3.3a and 3.3b. Figure 3.3b is a microscopic image of the area bounded by the dashed line in figure 3.3a. The width of the three inlets channels is $50 \mu \mathrm{m}$ while the outlet channel's width is $100 \mu \mathrm{m}$. At the cross junction the oil inlet channel tapers to $15 \mu \mathrm{m}$ and the two water inlet channels taper to $30 \mu \mathrm{m}$. The outlet channel begins at $30 \mu \mathrm{m}$ and quickly widens to $100 \mu \mathrm{m}$. The depth of all channels is $50 \mu \mathrm{m}$. Olive oil with $2 \%$ SPAM 80 surfactant was used as a continuous medium and water was used as a dispersed medium for injection into the cross junction. Oil was injected from the left side of the channel closest to the junction entrance (Inlet 2) by a syringe pump; water was pumped in from inlet 1 . The outlet channel was observed at three pre-defined detection points marked by A, B and C in figure 3.3a. The first detection point was close to the cross junction, the second further along the channel at the mid-point of the channel length $(0.5 \mathrm{~cm}$ from the junction) and the third point was close to the outlet reservoir ( $1 \mathrm{~cm}$ from the junction). 


\section{CHAPTER IV}

FORMATION OF NON-SPHERICAL DROPLETS AT CROSS JUNCTION

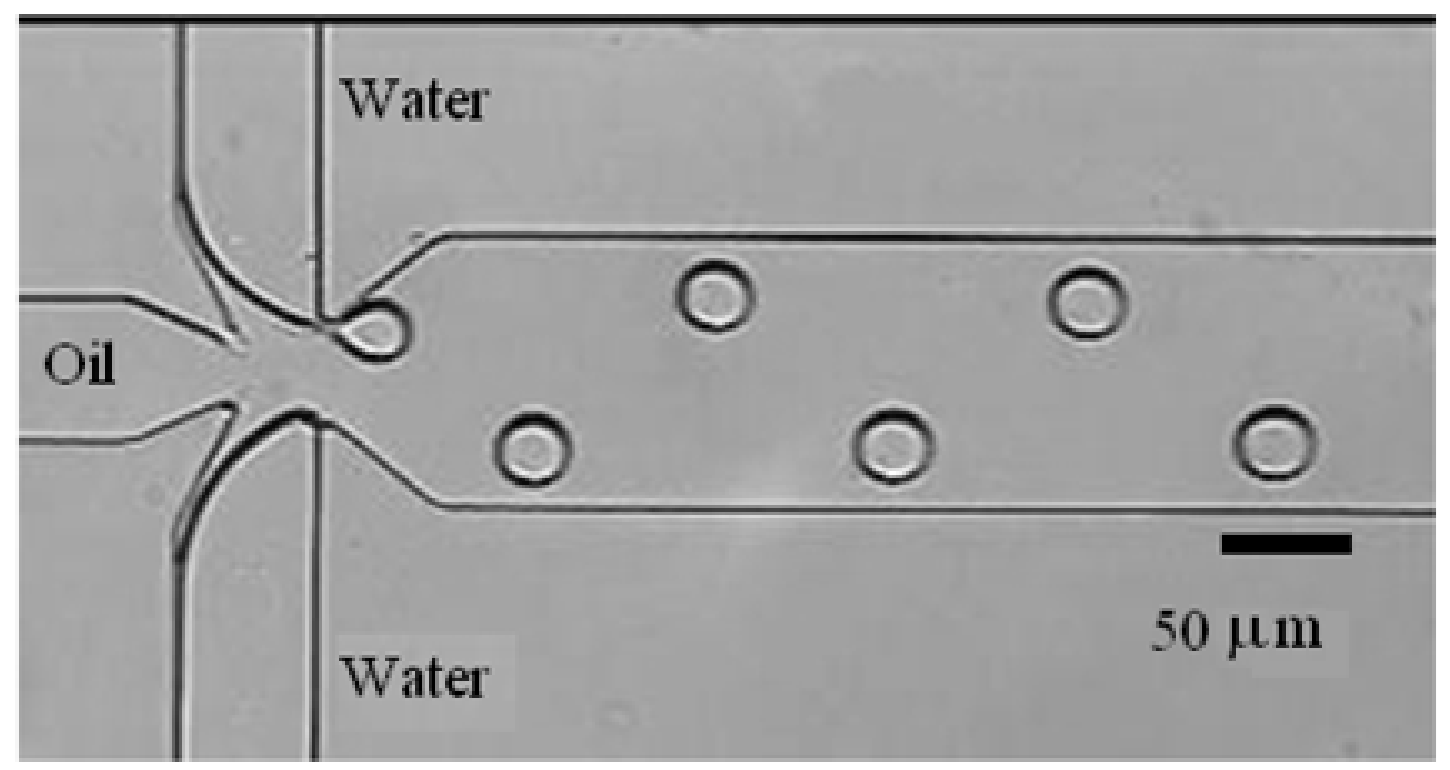

Fig. 4.1 Formation of droplets at the cross junction (detection point A). Oil flow rate $=$ $2.30 \mu \mathrm{L} / \mathrm{min}$, water flow rate $=0.20 \mu \mathrm{L} / \mathrm{min}$.

Figure 4.1 is a microscopic image that shows the formation of droplets at the cross junction (detection point A). Oil is being pumped at $2.30 \mu \mathrm{L} / \mathrm{min}$ and water at 0.20 $\mu \mathrm{L} / \mathrm{min}$. Droplets are produced periodically and alternatively from the two water channels. This is an important property of this design. No two droplets are formed at the same time. As each droplet is formed, a local pressure is built up in the oil medium around the junction which prevents the other opposing microchannel from forming a droplet at the same instant. Once the droplet is generated and flown into the main 
channel, the pressure built at the microchannel is released. Then, the other opposing microchannel starts producing the droplet. In this way no two droplets are formed simultaneously at the horizontally opposing two channels, instead we observe droplets being formed alternatively from each channel at periodic intervals.

A second property that was observed with these initial results is that the droplets tend to travel to the center of the channel. This phenomenon in shown in the images of figure 4.2.

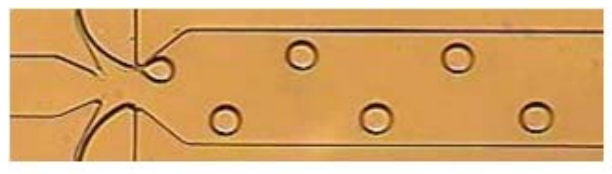

A

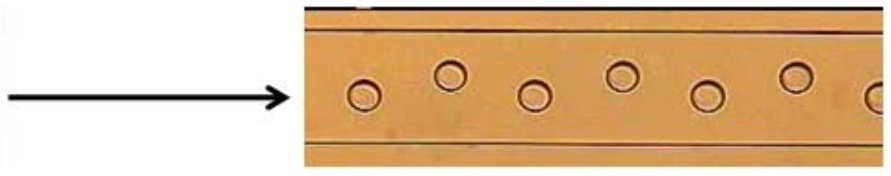

C

Fig. 4.2 Path of droplets in microchannel. Droplets tend to travel to the center of the channel. The image on the left was taken at detection point A (cross junction) and the image on the right was taken at detection point $\mathrm{C}(1 \mathrm{~cm}$ from the cross junction).

This property is a direct result of the velocity profile of a fluid traveling in a microchannel. Fluids display a parabolic velocity profile in microchannels (see figure 4.3). The velocity is highest at the center of the channel and lowest at the edges. 


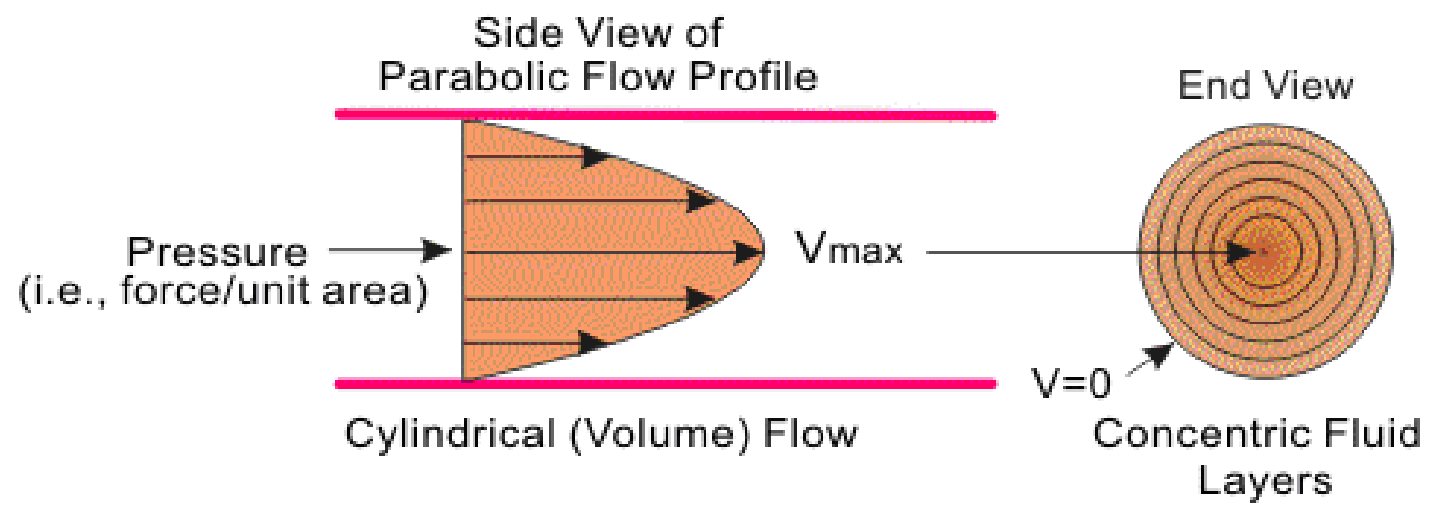

Fig. 4.3 Parabolic flow profile of microchannel.

This velocity profile also defines the pressure profile of the channel. The increase in velocity at the centre of the channel is accompanied with a decrease in pressure. Hence, the pressure is minimum at the center of the channel and maximum at the edges of the channels. This pressure difference across the width of the channels explains the path of the droplets. Droplets are formed closer to the walls of the channels but move towards the center where the pressure is lower and velocity is higher.

The droplets shown in figure 4.1 are spherical in shape and do not show any modification in shape as they travel. The droplets are approximately $20 \mu \mathrm{m}$ in diameter. The distance between the droplets is too large for these values of flow rates for the droplets to influence each others' shapes.

For the next set of readings the flow rate of oil was set to $2.00 \mu \mathrm{L} / \mathrm{min}$ and kept constant while the flow rate of water was steadily increased from $0.2 \mu \mathrm{L} / \mathrm{min}$ to 1.90 $\mu \mathrm{L} / \mathrm{min}$. If the water flow rate was reduced to below $0.2 \mu \mathrm{L} / \mathrm{min}$ the flow of water was cut off completely by the much higher flow of oil. Increasing water flow rate beyond 
$1.90 \mu \mathrm{L} / \mathrm{min}$ gave a continuous flow of water as water was able to overcome the shear force applied by oil flow.

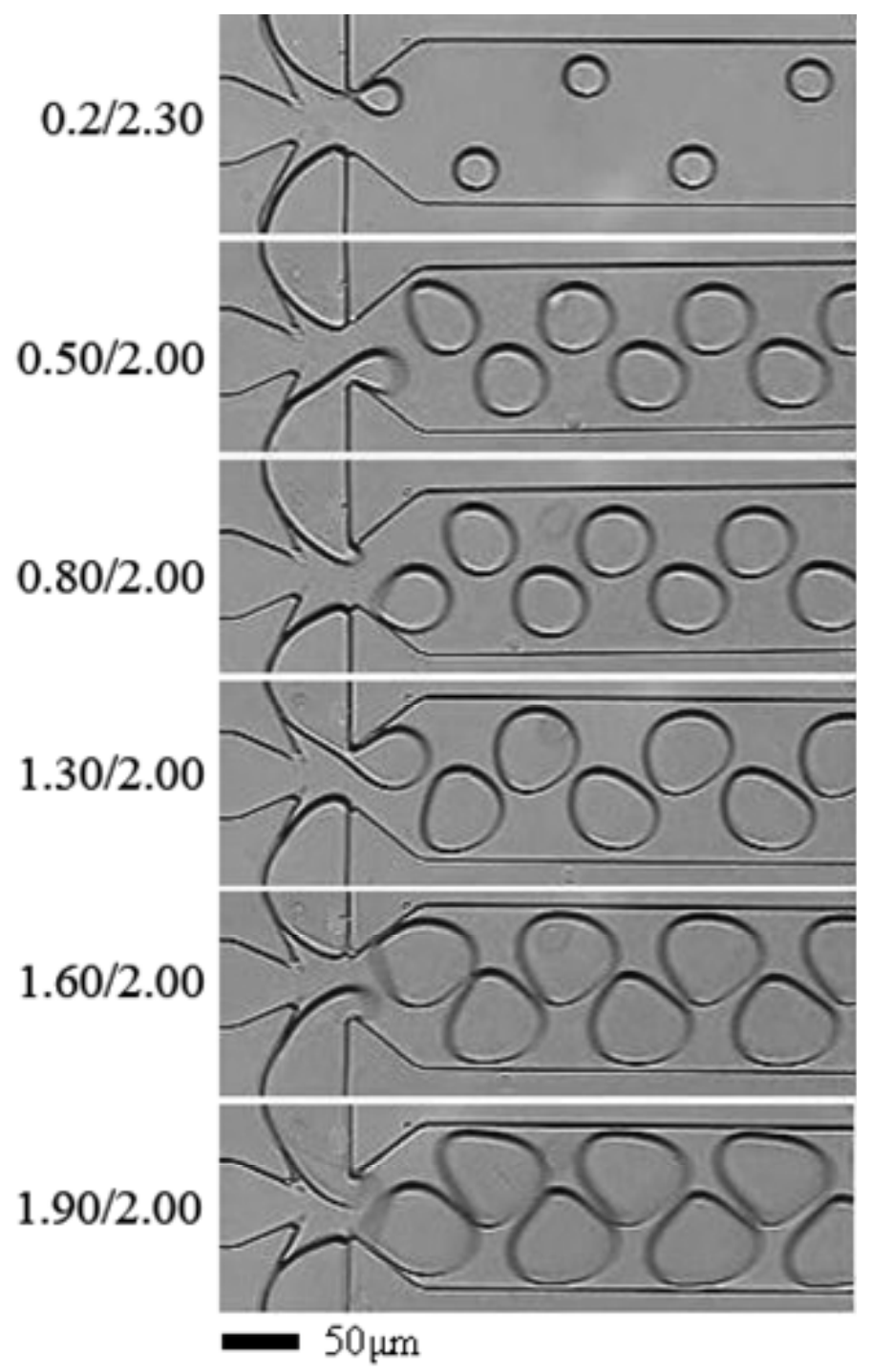

Fig. 4.4 Microscopic images of the cross junction (point A). These images show alternate generation of water droplets from both sides of water channel. The ratios are water flow rate to oil flow rate in $\mu \mathrm{L} / \mathrm{min}$. 
As can be seen in figure 4.4, the droplets are spherical in shape to begin with but their shape changes with increase in size. Small droplets are produced for small values of water flow rate. As the water flow rate is increased, and gets comparatively closer to the oil flow rate, the size of the water droplets increases. With this increase in size we observe that the droplets deviate from their spherical shape. The deviation continues to increase until the water flow rate overcomes the shear force and results in continuous flow of water and oil next to each other. As water flow rate is increased, the volume of water in oil (packing ratio of water droplets in oil media) increases, the distance between them decreases and the shape modification becomes more evident.

The shape change is derived from the device geometry and the flow rates. At low water flow rates there is enough distance between the droplets for them to take spherical shapes that occupy minimum surface area. As the water flow rate is increased and the pressure exerted causes more water per droplet to be pushed out the distance between the droplets decreases. This causes the droplets to line up next each other. Due to the slope at the point of droplet formation and the alternate ejection of droplets from each channel we see that the droplets have a wider surface facing towards the wall of the channel and a thinner surface facing the center. 


\section{CHAPTER V \\ DYNAMIC SHAPE MODIFICATION OF DROPLETS}

Figure 5.1 is a phase diagram that shows the modification of droplets at detection points $\mathrm{B}$ and $\mathrm{C}$ for various flow rate ratios. The first and third rows of images represent detection point $\mathrm{A}$ and the second and fourth rows represent detection point $\mathrm{B}$. As the water droplets get larger with increase in flow rate the shape modification becomes more evident. At water flow rate $0.5 \mu \mathrm{L} /$ minute we notice that droplets beginning to stretch out in the vertical direction as they move towards the outlet reservoir. Comparing this to microscopic images taken at higher water flow rate, it is observed that the stretching becomes more prominent until the droplet finally occupies the entire width of the microfluidic channel. The droplets continue to move in a periodic fashion and don't merge with each other. The change is reproducible and controlled by the flow rates. 


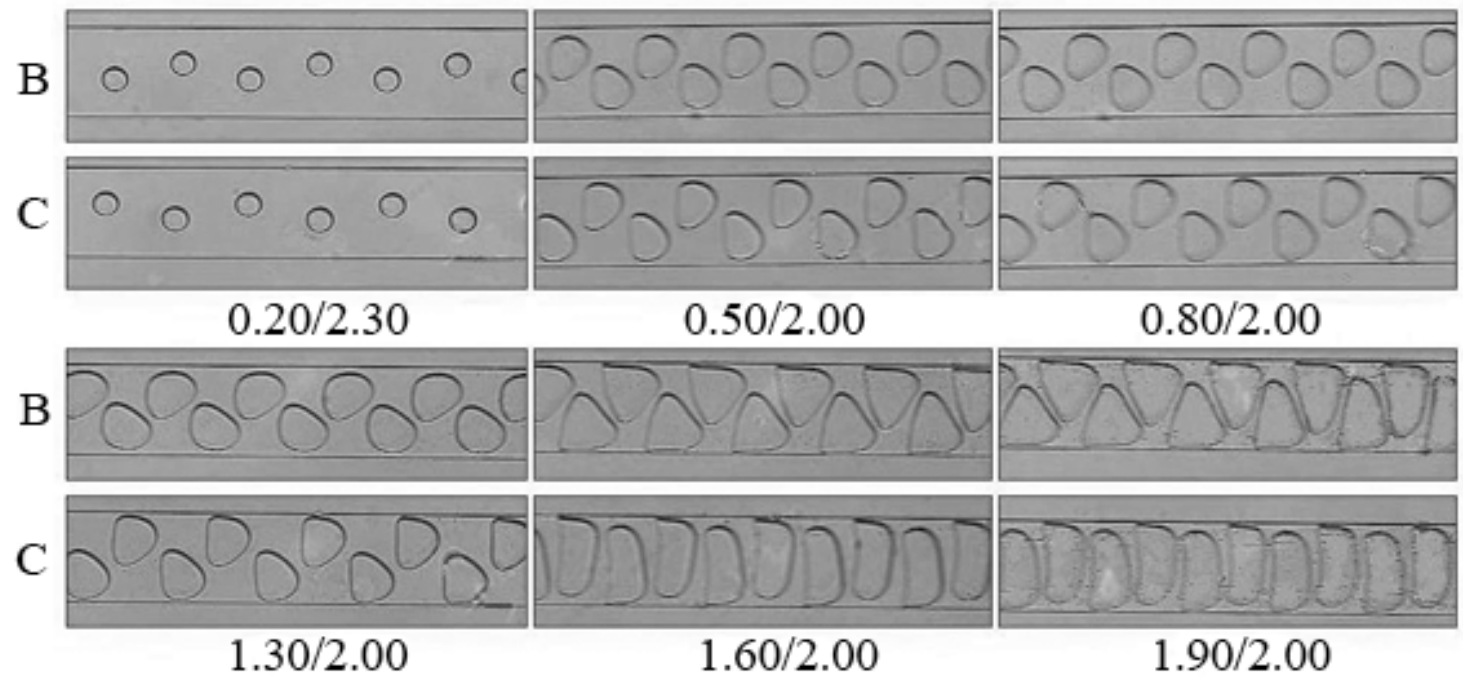

Fig. 5.1 Microscopic images of microchannel at detection points B and C at various flow rates. The points $\mathrm{B}$ and $\mathrm{C}$ are located at $0.5 \mathrm{~cm}$ and $1 \mathrm{~cm}$ away from cross junction respectively. The ratios are water flow rate to oil flow rate in $\mu \mathrm{L} / \mathrm{min}$.

The shape modification can be explained in terms of interfacial surface tension and pressure difference across the channel. Surface tension is caused by the attraction between molecules of a liquid by various intermolecular forces. Each molecule exerts an equal force on all molecules surrounding it. The molecules at the surface only have half the number of molecules surrounding them and exert an inward force which tends to minimize the surface area. Surface tension is expressed in terms of force per unit length or work done per unit area. For a given fluid's surface area to increase, work needs to be done on it which means change in the surface tension. According to the Young-Laplace equation:

$$
\left(\Delta \mathrm{p}=\gamma\left(\left(1 / \mathrm{R}_{1}\right)+\left(1 / \mathrm{R}_{2}\right)\right)\right.
$$


where $\Delta \mathrm{p}$ is the pressure difference sustained by the surface, $\gamma$ is the surface tension at the interface and $R_{1}$ and $R_{2}$ are the principal radii of curvature), if no normal force is acting on a surface then the surface must remain flat. However, if pressure on one side of the surface differs from pressure on the other side, a normal force acts on the surface which is equivalent to the pressure difference times the surface area. In order to balance the force acting on it, the surface tends to curve.

Due to the pressure difference across the width of the channel, various local pressure differences exist between the boundaries of the droplets. These local pressures cause the droplet to curve and stretch so that all forces acting on the droplet balance out. 


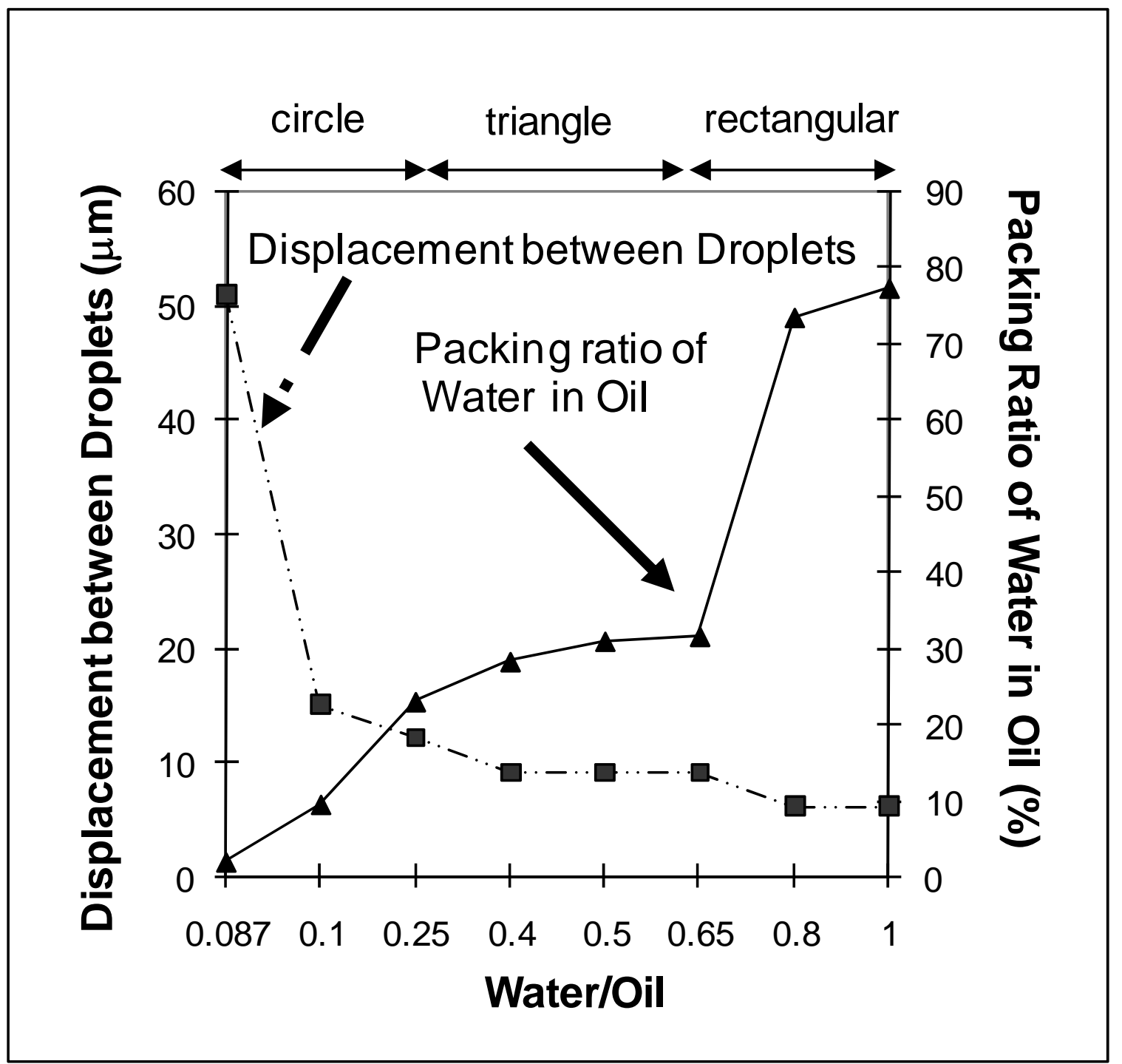

Fig. 5.2 Water droplet packing ratio in oil media and displacement between droplets as the function of water/oil flow rate ratio.

We were able to identify three different regimes for this set of readings that gave us distinct shapes. The shapes were identified as circular, triangular and rectangular, and 
their boundaries are shown in figure 5.2 which also tracks the displacement of droplets and the packing ratio of water in oil with change in water/oil flow rate ratio.

For the above set of readings we kept oil at a constant flow rate and changed the flow rate of water. The experiment was repeated for two more values of oil, $1.0 \mu \mathrm{L} / \mathrm{min}$ and $1.5 \mu \mathrm{L} / \mathrm{min}$. The results from all three sets are presented in figure 5.3.

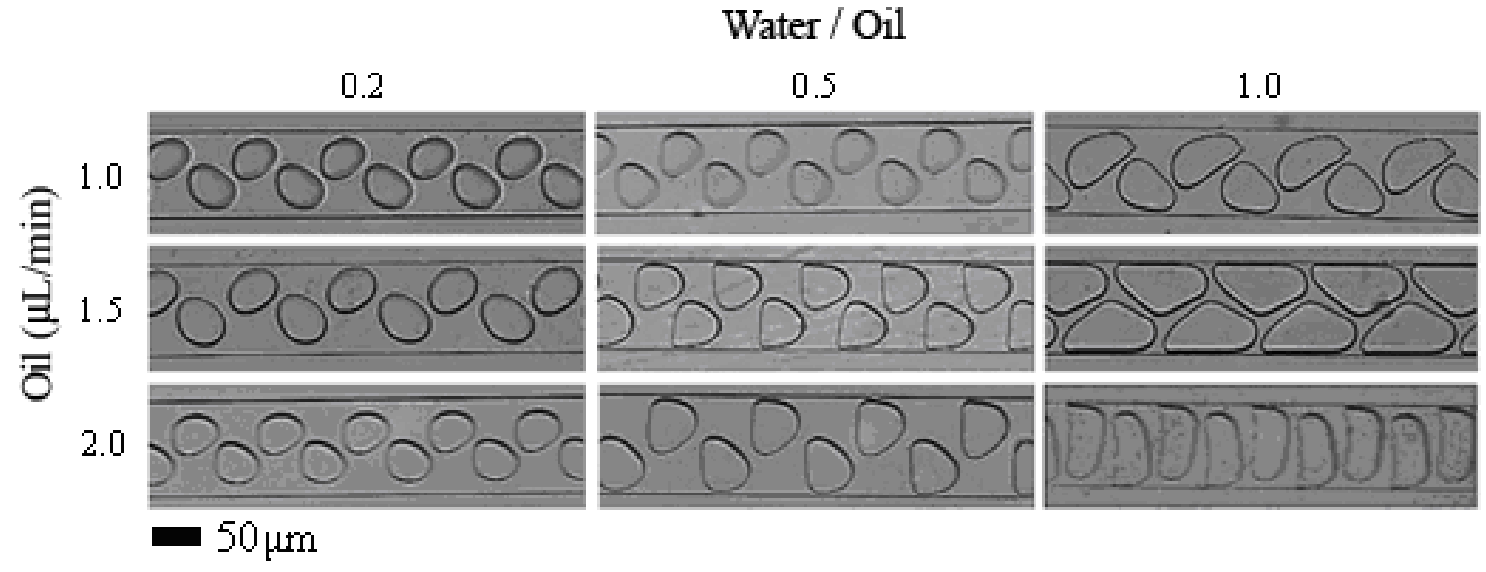

Fig. 5.3 Microscopic images of microchannel at detection point $\mathrm{C}$ at various flow rates. The ratios are water flow rate to oil flow rate in $\mu \mathrm{L} / \mathrm{min}$. 


\section{CHAPTER VI}

\section{CONCLUSIONS}

In conclusion, we have demonstrated a new application of microchannels which enables to produce polygonal droplets. A cross junction has been used which where water droplets are generated in an oil medium by shear forces.

Droplets are observed to spherical in shape when the flow rate ratio of water to oil is small. As this ratio increases and gets closer to 1 , the volume of water droplets increases. The distance between consecutive droplets reduces and the pressure difference across the width of the channel causes the droplets to lose their spherical shape. Nonspherical droplets are produced that were classified in to three regimes; circular, triangular and rectangular.

It has been shown that the droplets dynamically change their shape in transport in microchannels. The modification is controlled by process parameters such as flow rates and channel geometry. The dynamic modification has been explained in terms of interfacial surface tension and local pressure difference. Because of high packing rate of water droplet in oil media, these polygonal droplets may be utilized for high throughput molecular screening.

Future work can be pursued by using different geometries to try and create new shapes that were not created using this design. 
These results give us better control over the shape of droplets in microchannel that has been achieved before. They give us further insight in to the behavior of droplets in microchannels. 


\section{REFERENCES}

1 A. R. Wheeler, W. R. Throndset, R. J. Whelan, A. M. Leach, R. N. Zare, Y. H. Liao, K. Farrell, I. D. Manger and A. Daridon, Anal.Chem., 2003, 75, 3581-3586.

2 E. Y. Chan, N. M. Goncalves, R. A. Haeusler, Amie J. Hatch, J. W. Larson, A. M. Maletta, G. R. Yantz, E. D. Carstea, M. Fuchs, G. G. Wong, S. R. Gullans and R. Gilmanshin, Genome Res., 2004, 14(6), 1137-1146.

3 H.A. Stone, A.D. Stroock, and A. Ajdari, Annu. Rev. Fluid Mech., 2004, 36, $381-411$.

4 J. Khandurina, T. E. McKnight, S. C. Jacobson, L. C. Waters, R. S. Foote, and J. Michael Ramsey, Anal. Chem., 2000, 72, 2995-3000.

5 D. Figeys, S. P. Gygi, G. McKinnon and Ruedi Aebersold, Anal. Chem., 1998, 70 (18), 3728-3734.

6 D. L. Stokes, G. D. Griffin and T. Vo-Dinh, Fresenius J. Anal. Chem., 2001, 369, $295-301$.

7 D. A. LaVan, T. McGuire and R. Langer, Nat. Biotech., 2003, 21(10), $1184-$ 1191.

8 Petra S. Dittrich and Andreas Manz, Lab Chip, 2006, 5, 210-218.

9 M. A. Burns, B. N. Johnson, S. N. Brahmasandra, K. Handique, J. R. Webster, M. Krishnan, T. S. Sammarco, P. M. Man, D. Jones, D. Heldsinger, C. H. Mastrangelo and D. T. Burke, Science, 1998, 282, 484-487. 
10 M. Joanicot and A. Ajdari, Science, 2005, 309, 887-888.

11 D. J. Beebe, G. A. Mensing, and G. M. Walker, Annu. Rev. Biomed. Eng., 2002, 4, 261-286.

12 H. Song, J. D. Tice and R. F. Ismagilov, Angew. Chem. Int. Ed., 2003, 42, 768772.

13 D. Dendukuri, K. Tsoi, T. A. Hatton and P. S. Doyle, Langmuir, 2005, 21, $2113-$ 2116.

14 K. Ramser, J. Enger, M. Goksor, D. Hanstorp, K. Loggb and M. Kall, Lab Chip, 2005, 5, 431-436.

15 P. Garstecki, M. A. Fischbach and G. M. Whitesides, Appl. Phys. Lett., 2005, 86, 244108.

16 T. Nisisako, S. Okushima and T. Torii, Soft Matter, 2005, 1, 23-27.

17 P. Marmottant and S. Hilgenfeldt, Proc. Natl. Acad. Sci. USA, 2004, 101(26), 9523-9527.

18 V. Srinivasan, V. K. Pamula and R. B. Fair, Lab Chip, 2004, 4, 310-315.

19 B. Zheng, J. D. Tice and R. F. Ismagilov, Adv. Mater., 2004, 16, 1365-1368.

20 M. Prakash and N. Gershenfeld, Science, 2007, 315, 832-835.

21 J. D. Tice, H. S., Adam D. Lyon, and R. F. Ismagilov, Langmuir, 2003, 19, $9127-9133$.

22 T. Nisisako and T. Torii, Adv. Mater., 2007, 19, 1489-1493.

23 D. Dendukuri, D. C. Pregibon, J. Collins, T. A. Hatton and P. S. Doyle, Nat. Mater., 2006, 5, 365-369. 
24 G. M. Whitesides and A. D. Stroock, Phy. Today, 2001, 54, 42-48.

25 D. Klotzkin and I. Papautsky, SPIE, 2007, 0748, DOI: 10.1117/2.1200705.0748.

26 Y. Tan, J. S. Fisher, A. I. Lee, V. Cristini and A. P. Lee, Lab Chip, 2004, 4, 292298.

27 P. Garstecki, M. J. Fuerstman, H. A. Stone and G. M. Whitesides, Lab Chip, 2006, 6, 437-446.

28 T. Nisisako, T. Torii and T. Higuchi, Lab Chip, 2002, 2, 24-26.

29 S. Okushima, T. Nisisako, T. Torii, and T. Higuchi, Langmuir, 2004, 20 (23), 9905-9908.

30 S. Hsiung, C. Chen and G. Lee, J. Micromech. Microeng., 2006, 16, 2403-2410.

31 Y. Tan and A. P. Lee, Lab Chip, 2005, 5, 1178-1183.

32 S. Abraham, E. H. Jeong, T. Arakawa, S. Shoji, K. C. Kim, I. Kim and J. S. Go, Lab Chip, 2006, 6, 752-756.

33 J. H. Xu, G. S. Luo, S. W. Li and G. G. Chen, Lab Chip, 2006, 6, $131-136$.

34 S. L. Anna, N. Bontoux and H. A. Stone, App. Phy. Letters, 2003, 82, 364-366. 


\section{VITA}

Rajat Mehrotra received his Bachelor of Science degree in electrical engineering from Texas A\&M University at College Station in 2006. She entered the Master of Science program at Texas A\&M University in September 2006 and received her Master of Science degree in May 2008.

Mr. Mehrotra may be reached at Department of Electrical and Computer Engineering, 214 Zachry Engineering Center, Texas A\&M University, College Station, TX-77843. His email address is mehrotra@tamu.edu. 